\title{
Towards a systems approach for chronic diseases, based on
}

\section{health state modeling [version 1; peer review: 3 approved, 1}

\section{approved with reservations]}

\author{
Michael Rebhan (iD
}

Novartis Institutes for Biomedical Research, Basel, 4056, Switzerland

V1 First published: 23 Mar 2017, 6:309

https://doi.org/10.12688/f1000research.11085.1

Latest published: 23 Mar 2017, 6:309

https://doi.org/10.12688/f1000research.11085.1

\section{Abstract}

Rising pressure from chronic diseases means that we need to learn how to deal with challenges at a different level, including the use of systems approaches that better connect across fragments, such as disciplines, stakeholders, institutions, and technologies. By learning from progress in leading areas of health innovation (including oncology and AIDS), as well as complementary indications (Alzheimer's disease), I try to extract the most enabling innovation paradigms, and discuss their extension to additional areas of application within a systems approach. To facilitate such work, a Precision, P4 or Systems Medicine platform is proposed, which is centered on the representation of health states that enable the definition of time in the vision to provide the right intervention for the right patient at the right time and dose. Modeling of such health states should allow iterative optimization, as longitudinal human data accumulate. This platform is designed to facilitate the discovery of links between opportunities related to a) the modernization of diagnosis, including the increased use of omics profiling, b) patientcentric approaches enabled by technology convergence, including digital health and connected devices, c) increasing understanding of the pathobiological, clinical and health economic aspects of disease progression stages, d) design of new interventions, including therapies as well as preventive measures, including sequential intervention approaches. Probabilistic Markov models of health states, e.g. those used for health economic analysis, are discussed as a simple starting point for the platform. A path towards extension into other indications, data types and uses is discussed, with a focus on regenerative medicine and relevant pathobiology.

\section{Keywords}

chronic diseases, systems approach, Precision Medicine, computational modeling, disease progression, Markov health state models, Regenerative Medicine, Open Science.

\section{Open Peer Review \\ Approval Status $\checkmark \checkmark \checkmark$ $\begin{array}{llll}1 & 2 & 3 & 4\end{array}$ version 1 23 Mar 2017 view view view view \\ 1. Michel Goldman (D), Université Libre de Bruxelles, Brussels, Belgium \\ 2. Rob Hooft van Huijsduijnen (iD), Independent consultant, Geneva, Switzerland \\ 3. Jacques S. Beckmann ID, Université de Lausanne, Lausanne, Switzerland \\ 4. Charles Auffray ID , Université de Lyon, Lyon, France Bertrand De Meulder (iD), Université de Lyon, Lyon, France \\ Manlio Vinciguerra, International Clinical Research Center (FNUSA-ICRC), Brno, Czech Republic}

Any reports and responses or comments on the article can be found at the end of the article. 
Corresponding author: Michael Rebhan (michael.rebhan@novartis.com)

Competing interests: The author is an employee of the research organization of a pharmaceutical company (Novartis Pharma AG, Basel, Switzerland). The author acknowledges a bias towards OpenScience principles, as outlined above, which may not reflect the mainstream mindset in his industry.

Grant information: My employer (Novartis Institutes for Biomedical Research) funded the work on this text.

Copyright: @ 2017 Rebhan M. This is an open access article distributed under the terms of the Creative Commons Attribution License, which permits unrestricted use, distribution, and reproduction in any medium, provided the original work is properly cited. Data associated with the article are available under the terms of the Creative Commons Zero "No rights reserved" data waiver (CC0 1.0 Public domain dedication).

How to cite this article: Rebhan M. Towards a systems approach for chronic diseases, based on health state modeling [version 1; peer review: 3 approved, 1 approved with reservations] F1000Research 2017, 6:309 https://doi.org/10.12688/f1000research.11085.1

First published: 23 Mar 2017, 6:309 https://doi.org/10.12688/f1000research.11085.1 


\section{Rising pressure from chronic diseases}

One of the main challenges our healthcare and biomedical research and development systems are facing, in the age of digitalization and aging populations, is a rising burden from chronic conditions. This burden has a multitude of effects not only on the Quality of Life (QoL) and well-being of the patients and their immediate social networks (e.g. family members), but it also triggers increasing discussion about sustainability problems in health-related systems, including the economics of healthcare systems. Medical conditions are defined as being 'chronic' when they last 12 months or more, result in functional limitations (which tend to reduce QoL) and/or the need for ongoing medical care (i.e. healthcare resource utilization). Costs associated with chronic conditions are on the rise in many countries, and have been identified as a main driver of medical cost explosion, leading into the economic sustainability discussion. By now, they cause the majority of all healthcare costs in developed countries, with fastrising prevalence in some emerging countries as well, as their societies increasingly imitate developed countries, including lifestyle, economy and burden from chronic diseases.

For example, in the US, a country that is among the most advanced in terms of this development, $31.5 \%$ of the population in 2010 was affected not only by a single, but multiple chronic conditions (MCC), binding more than $70 \%$ of all healthcare spending (not considering other costs, outside healthcare budgets, such as social care) (Gerteis et al., 2014). Chronic diseases overall, including patients with a single chronic condition, account for a vast majority $(86 \%)$ of healthcare spending in the US (Gerteis et al., 2014), leading to intensive discussion on how long society can afford to pay for rising healthcare budgets (Callahan, 2013), which are based on economic models that are largely disconnected from outcomes achieved (EFPIA, 2015). In terms of indications, metabolic (e.g. diabetes), cardiovascular (e.g. heart disease), respiratory (e.g. COPD and asthma), autoimmune (e.g. rheumatoid arthritis), and neurological conditions (e.g. Alzheimer's, and Parkinson's disease) are typically among the most commonly observed, depending on the country and population (Callahan, 2013; Gerteis et al., 2014; Nugent, 2008; and Kvedar et al., 2016; see also the Global Burden of Disease study below).

This increase in chronic diseases in both developed and also emerging countries (Nugent, 2008) represents a challenge that forces us to go back to the design board, in terms of the healthrelated systems we have created, to increase their ability to cope with what's growing in terms of challenges. As a recent article that explains the need for such a fundamental redesign puts it: we face a "critical turning point, requiring not only improved health care systems but also a new model of medicine at its foundation" (Callahan, 2013). Similar statements can be found in the discourse of other disciplines involved in health innovation, including biomedical research and its translation (Butler, 2008; Cooksey, 2006; Lazebnik, 2002; Munos, 2010; Munos, 2016; Poste, 2011). At the same time, due to medical progress in specific areas, some of the diseases that were almost impossible to survive a while ago, now turn into new types of chronic conditions, e.g. AIDS (where personalized combination therapies have enabled impressive improvements of patient outcomes in a relatively short time, see below). Such new chronic conditions created by medical progress also require sustained care and resources over many years, further increasing chronic disease burden. This trend of medical innovation creating new chronic conditions is likely to continue. "It is now possible, and not uncommon, for someone to have cancer pushed into remission at 65 , to persist with well-managed heart disease at 75, and then to acquire Alzheimer's at 85" (Callahan, 2013). Therefore, the rise in life expectancy that follows increasing development according to the Western model of modernization of the last $2-3$ centuries is accompanied by more time spent in a managed chronic condition. This, in turn, leads to a lively debate on the need to push innovation for 'healthy aging', considering not only how long we live, but also the QoL of those added years. In that context, what can we learn about 'healthy aging', in the absence of a heavy burden from chronic diseases, in populations that do better than average?

\section{Islands of healthy aging}

Comparisons between different human populations (e.g. in different geographies, or between subpopulations that live in the same geographic area) can reveal interesting patterns related to this debate. Studies of human populations that enjoy both a long and healthy life compared to others in their proximity (i.e. "islands of healthy aging"), so far have revealed that there are candidate contributing factors for healthy aging at many levels, including genetics, various aspects of lifestyle, environmental context, sociology and culture, and of course economic factors. However, it is important to be cautious about accepting simplified conclusions from such studies, as they suffer from the same fundamental problems as other types of studies in complex human populations, including the risk of unintentionally comparing apples and oranges (which can reveal the wrong factors as being significant), as well as the temptation of jumping from correlations to statements on causation (as it often happens in the mass media, which adds to widespread confusion on the topic).

In the case of the so-called 'Blue Zone' populations of central Sardinia (Pes et al., 2013), a Mediterranean island with pleasant climatic conditions, various studies aim to identify significant differences between the healthy aging 'Blue Zone' populations, which are known as some of the most long-living populations in the Western world, and other Sardinian populations that have a closeto-average life expectancy and health profile during aging. Note that the 'Blue Zone' populations in the center of the island are known to have been slower in adopting a modern lifestyle, compared to the people on more accessible coastal areas (a pattern that can be observed in similar landscapes, where accessibility of geographies influences speed of modernization). A statistical analysis of factors that clearly distinguish both Sardinian populations from each other (i.e. Blue Zone populations from the others) revealed occupational aspects (with communities rich in shepherds being healthier than those with more farmers and fishermen), landscape (mountainous terrain being a healthier environment compared to coastal lowlands), and dietary factors (with Barley production associated with healthy aging) as significant. A possible conclusion from such an analysis of correlations could be that healthy aging populations are rather found in areas with many shepherds, who used to spend much time roaming sparsely populated, mountainous areas, and 
less than in areas with more intensive spurts of activity typical for farmers and fishermen. Other conclusions may be valid as well, and it can be difficult to choose among the alternative conclusions, to inform action.

Based on our current knowledge about the characteristics of healthy aging populations, and risk factors for increased burden from chronic diseases (e.g. from the Framingham and similar longitudinal observational cohort studies; Mahmood et al., 2014), initiatives aimed at reducing chronic disease burden in public health have tried to develop solutions that work in an efficient manner at population level, including educational, political, regulatory and medical initiatives. One of the most visible exemplars for successful paradigms in public health is the reduction of burden associated with smoking and second-hand smoking reduction, highlighting the power of coordinated, interdisciplinary collaboration towards a higher-level health goal. In that context it is important though to point out that much of the evidence we have is, as stated above, only correlative in nature, and that its efficient reduction to practice in terms of the best (combination and/or sequence) of interventions in different populations and settings is anything but trivial (see also Carter, 2015, and the discussion on AIDS and platform applications below). For example, let's assume that many studies confirm that a shepherd-like lifestyle in mountains with mild climate, regular siesta and associated diet is indeed the one that gives us the most healthy aging experience, how do we extend such a 'successful lifestyle paradigm' into another setting that is less peaceful and traditional, e.g. a busy, modern, urban environment with its strong selective pressures on lifestyle and culture?

\section{Systems approaches}

With all the (somewhat fragmented) knowledge we have accumulated, and made increasingly accessible with digitalization, I propose that it is a good time to learn how to "put the pieces of the puzzle together", by learning how to best link and extend the most successful paradigms. Learning, in this case, means to understand the most powerful combinations of paradigms, where a paradigm can and where it cannot be applied (its 'domain of validity'), and what adjustments to its implementation are needed to fit a particular situation. Several examples are provided below, e.g. the lessons learned from the modernization of diagnosis in oncology and AIDS, combined with innovation on more personalized (combinatorial) interventions. To achieve this, we need to learn how to better connect relevant 'pieces of knowledge' and stakeholders, across disciplines, institutions and other real life barriers, towards increased speed and effectiveness of distributed learning, at systems and community level. This should put following generations into a better position for managing not only problems related to sustainability in health, which our generation is still struggling with, but also problems in other (connected) areas that pose similar challenges.

However, if we do a reality check, of our status quo, this is the type of challenge we, at our current stage of human cultural evolution, have provided only limited evidence so far for actually being able to cope with. Several thousand years after a series of cultural transitions from small communities of hunter-gatherers (with a more limited control over their environment) into increasingly large and complex, globally connected societies (with more widespread effects in our environment, including the most remote corners of our planet), the question poses itself: what is the next stage in our cultural evolution, as a species? Will it actually be possible to overcome obstacles on the path towards multi-stakeholder co-design of healthier and more sustainable systems, and how long will it take?

In the life sciences, including fields related to medicine and biology, we can find many good initiatives that point into this direction, but also a widespread disbelief among leaders in those disciplines that we will be able to fundamentally change things, because "things that never change" (which translates into the implicit belief that we have reached the end of human cultural evolution, in terms of our ability to manage certain types of complexity, as a human population) and the special characteristics (complexity) of living systems compared to engineered systems (e.g. see Lazebnik, 2002). Good introductions into those important discourses, in the above context, are provided by

- Altman, 2012 (linking the molecular and clinical worlds; role of systems medicine)

- Auffray et al., 2016 (focus on European initiatives, and the need to connect those)

- Barker, 2011 (sustainability of healthcare systems, with US and UK focus)

- Butler, 2008 (the 'valley of death' problem, translating innovation to impact)

- Callahan, 2013 (sustainability of medicine, healthy aging, chronic diseases)

- Carter, 2015 (healthy aging, medical philosophy, and public health policies)

- Goodwin, 1999 (evolution of science, from control to participation)

- Koelsch et al., 2013 (economic sustainability of personalized health model in Oncology)

- Lazebnik, 2002 (blind spots in biomedical research, lack of common language)

- Mathews \& Pronovost, 2011 (need for better systems integration in medicine)

- Munos, 2010 (from a non-sharing, competitive culture to open science in biomedical R\&D)

- Munos, 2016 (innovation crisis in pharma R\&D, and economic sustainability of the industry)

- Poste, 2011 (problems related to biomarkers and diagnostics)

- Powell, 2004 (systems approaches in biology, key problems and some trends)

- Pritchard et al., 2017 (translating PM into regular clinical care, key challenges, adoption) 
The ability to make progress here requires an increased capability for understanding how different aspects in relevant subsystems influence each other dynamically. Recently, we can observe early signs of a transition to a "new health innovation ecosystem' with changes in many subsystems, based on changing roles (e.g. of patients, physicians and pharmacists), processes, habits, and underlying economic models (Barker, 2011; Beckmann \& Lew, 2016; Koelsch et al., 2013; Munos, 2010), as a first symptom of efforts to increase systemic sustainability, as well as the effects of technology advances (see below). The types of challenges we need to tackle includes the need for a discourse of important tradeoffs that require careful balancing of the perspectives of multiple stakeholders. For example, as we develop therapeutic solutions for increasingly smaller but more molecularly defined populations in oncology, based on diagnostic modernization (see below), this results in tension between high prizes for targeted therapies and the enormous investment required to develop new targeted therapies in a highly regulated, and cost-intensive industry (Koelsch et al., 2013; Kostic \& Phillips, 2015?). Another fundamental trade-off situation with many consequences for a variety of stakeholders can be found when considering the resources currently involved in the last 5-10 years of life, including elderly, health, social and other care (e.g. by family members). Is a move towards more robots taking care of our elderly the only solution we can imagine, since there will not be enough people around to provide more human versions of care? Resolving such and other interconnected, multi-stakeholder challenges with trade-off tensions in their core will be an important set of problems to address, see Figure 1. In this context, a renewed and more widespread interest in systems approaches as a tool for managing such complexity is on the cards. For a brief overview of potentially relevant fields, concepts and tools related to systems approaches, see Box 1.

\section{Box 1. Expertise and tools related to systems approaches}

In this article, I define systems approaches as efforts aimed at 'connecting the pieces of the puzzle', i.e. a set of connected parts or subsystems (i.e. system components) that influence each other, with an emphasis on understanding the interactions between those parts, and how they contribute to system-level properties. System-level properties include emergence, which means that the system displays behaviors that depend on the way how the system components interact with each other, and robustness, a property that captures the ability of a system to deal with changes in its environment (e.g. living systems have evolved a collection of complementary system motifs that enhance their ability to cope flexibly with changes of food supply). Systems approaches can build on knowledge and tools from a range of fields, such as systems science, complexity theory, computational modeling of complex natural systems (e.g. in ecology and economy), nonlinear systems theory, self-organizing systems, chaos theory, cybernetics, whole systems thinking, general systems theory, and game theory. Introductory texts into some of the most relevant fields, their key concepts and tools, can be found in Goodwin (1999); Hammond (2005); Lazebnik (2002); Powell (2004); Sterling (2003); and Bousquet et al. (2011).

For most of the $19^{\text {th }}$ and $20^{\text {th }}$ century, our mindset was preoccupied with certain ideas of 'development' and 'civilization', with mostly negative views of other lifestyles found in 'less developed' areas, and a belief in a core role of new technologies to enable even more development towards an even better civilization. As such development, spreading globally, led to increasing awareness about the "other side of the coin", i.e. negative consequences for human and non-human species, this fueled excitement on finding better ways to understand complex systems that involve living species, e.g. how effects related to human development (e.g. pollution,

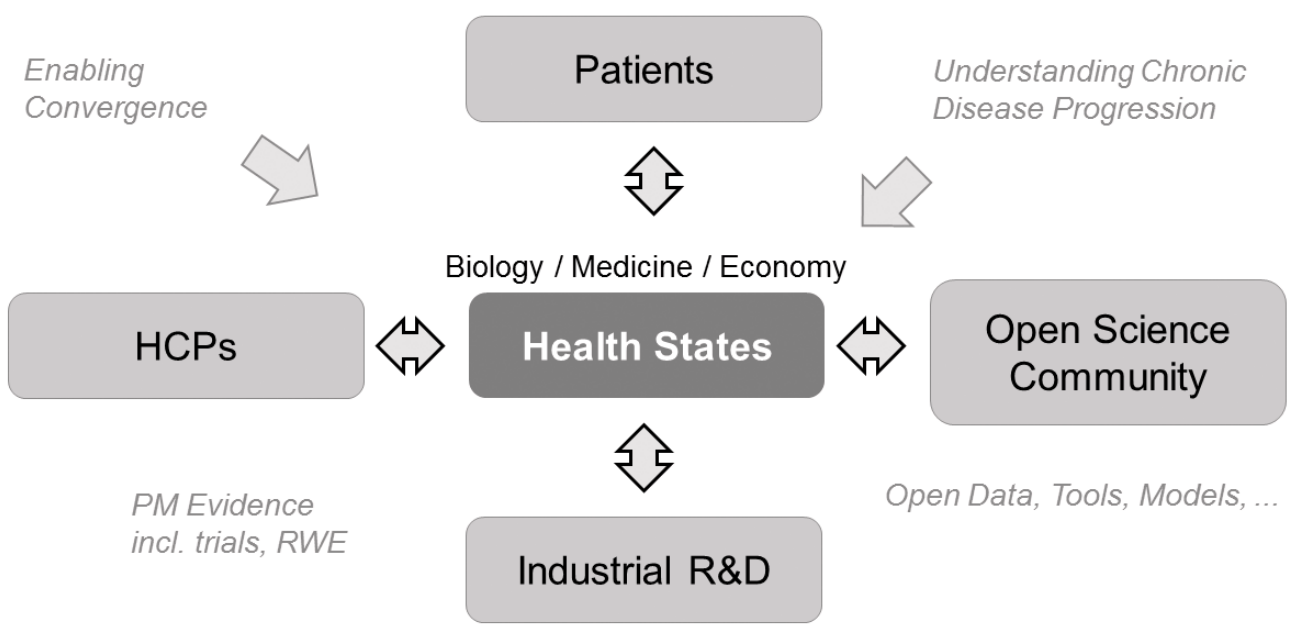

Figure 1. The proposed innovation ecosystem for chronic diseases, with a new platform that engages different health innovation stakeholders, and allows the emergence of interdisciplinary understanding of health states across biology, medicine and health economics in its digital center. The design is based on the ambition that all stakeholders should benefit from the development of this digital center. RWE = real world evidence. 
change of environments, increasing density of human populations, waste) affected the health of ecosystems (e.g. lakes undergoing eutrophication based on system shifts, with deadly consequences for the species that used to inhabit this biosphere; Yang et al., 2008). Over time, such 'ecosystem'-related fields developed the capability to understand recurring principles in that complexity, including the role of the connectivity between individual system components (Sterling, 2002). Interestingly, this revealed common patterns found in many complex systems, adding further fuel to the interest in systems approaches as a tool for managing complexity.

The ability to perform experiments, and the use of increasing computational power to develop better in silico models, at systems level, played an important role in this process. However, an early attempt to apply developments in those areas to molecular networks involved in disease, in the form of 'systems biology' (Lazebnik, 2002; Powell, 2004; Spivey, 2004), got slowed down by a few fundamental challenges. The effort and time needed to advance our understanding of all relevant system components and their interactions in human health, at sufficient detail for determining the best intervention (i.e. 'target') for promoting a transition to a particular health state, is immense, and there is doubt if that vision can even be achieved despite technology advances (e.g. omics technologies that can monitor the state of thousands of such molecules in living systems, see below). As a consequence of this 'cool down' on systems biology (enabled by omics profiling), many academic researchers in the field have shifted, over the years, to study simpler systems that are more remote from human complexity first, e.g. (populations of) easier-to-study single cell organisms with more simple genomes and behaviors, while applied research and medical innovation in industry largely focuses on other paradigms for generating starting points for their innovation pipelines, e.g. based on the screening of biological systems that model selected aspects of disease (to find starting points for new therapies). Note that institutes designed around a long-term investment into systems biology approaches, such as Lee Hood's Institute of systems biology in Seattle, have made considerable contributions to the continued discourse on the need for systems approaches in health innovation, and the development of guiding principles for P4 Medicine (e.g. Bousquet et al., 2011; Hood et al., 2014, and below). However, this discourse if by now substantially different from the ambitions of the systems biology wave about 10 years ago, as the community was getting excited about a new ability to 'know all the parts' and put the picture together on their interactions.

At the same time, there is increasing recognition that 'biomarkers' may become important 'anchors' in those complex networks, due to the ability to study their links with medical, economic and other non-biological data related to chronic diseases, including connections between diagnosis and intervention (see below). Scientific discussions related to this shift towards biomarkers (Burns et al., 2013; Poste, 2011) are one of the origins of the proposed platform. They may also serve as useful scientific bridges between key stakeholders (Figure 1), e.g. between different Intellectual Property/innovation domains such as the OpenScience community (where their efforts add information on the role of biomarkers and biomarker-based health state models) vs. proprietary therapeutic assets in pharmaceutical R\&D pipelines (e.g. where mechanism of action biology of those assets connects with such biomarkers, and health state biology). Similar issues may occur at interfaces between patient/consumer-centric solutions (e.g. through digital health), and those deployed in hospitals (i.e. for health care providers, HCPs), see Figure 1, with their different Intellectual Property/innovation domains. Biomarkers, as they contribute to the development to the interdisciplinary understanding of health states across stakeholders, are therefore an important focus of the proposed systems approach.

Of particular interest, from a systems point of view, will be knowledge related to the ability of different kinds of systems to cope with external changes (i.e. system robustness), including pressures outside the normal range of what the system is typically encountering (short time scales), or what it was encountering during its evolution (longer time scales). In a time of complex interactions between changes in various fields related to chronic diseases, we need to understand more about what makes systems robust despite change, and how the forces that drive change, and their effects, are connected. Kuhn's thoughts on recurring, cyclic patterns in the history of science that he called 'paradigm shifts' (Kaiser, 2012), including the accumulation of 'anomalies' that are inconsistent with the dominating paradigm(s), may be helpful. More widespread adoption of tools related to systems approaches, outside the existing, rather small group of experts, in areas where theory and practice collide for better learning, will be an enabling development for the proposed platform. In that context, it is important to develop a modeling-based learning process in the public domain, on a neutral platform that involves many stakeholders.

\section{Relationship with Precision, P4 and Systems Medicine}

As different aspects of an emerging consensus on how to develop more sustainable health-related systems are discussed in the literature and other media, due to the early stage of the discussion a variety of terms that capture key elements of the transition are used with inconsistent meaning, adding to overall confusion. Some of the terms that try to capture the ambitions of a 'new health innovation ecosystem', range from 'Precision Medicine', 'Personalized Health' to 'P4 Medicine ( $\mathrm{P} 4$ because of the four principles, starting with 'p': predictive, personalized, preventive, participatory)' and 'Systems Medicine'. For a recent overview on this discourse, see Auffray C et al., 2016; Bousquet J et al., 2011; Flores et al., 2013; Hawgood et al., 2015; Hood et al., 2014; Hood \& Price, 2014; Kodrič K et al., 2016; Kostic \& Phillips, 2016; Scholz, 2015; Wang et al., 2015; and Wilckens T, 2016. Comparison with the guiding principles of evidence-based medicine is provided by Beckmann \& Lew, 2016. Going forward, I will use the simplified abbreviation 'PM', as it captures at least some of the more commonly used terms (i.e. Precision/Personalized/P4 Medicine) in a simple abbreviation, assuming that systems approaches are an important tool on the path to the development of sustainable PM-based systems. The proposed platform is designed in a way that can accommodate the early stage of the emerging consensus in PM, and facilitate its maturation. 


\section{Aims of this article}

In this article, I aim to make a contribution to this discourse by 1) discussing potentially reusable, successful paradigms from selected areas of medical innovation, 2) leading to guiding principles for designing a platform that enables multi-stakeholder initiatives, centered on a theory of health states. In terms of interdisciplinary interfaces, our focus is on connections between medicine, biology and economy, and initially focus on applications related to regenerative medicine. Iterative optimization of the proposed reference health state models would be fueled by linking opportunities related to a) the modernization of diagnosis, b) ability to capture health state profiles using omics, c) patient-centric approaches based on technology convergence, d) increasing understanding of the pathobiology, clinical meaning and health economic aspects of disease progression stages, and e) design of new interventions, including therapies as well as preventive measures.

\section{Successful paradigms, from leading areas of health innovation}

Looking across different areas of medicine, we can notice interesting differences, e.g. in sharing culture, commonly applied tools, mindsets and approach, affecting the translation of advances in knowledge into improved patient outcomes, as well as the generation of new advances that fuel further progress. Here, I would like to highlight a number of successful paradigms with an impact on patient outcomes, and their potential relevance in the above discourse, even outside the problem areas in which they were originally developed.

Modernization of diagnosis, and personalization of therapy In discussions on the "valley of death" challenge in health innovation (Butler D, 2008; Wehling, 2009), which concerns the problem of translating scientific and technical advances into impact at the level of patient outcomes, beyond time-limited clinical studies, oncology is often mentioned as an area of medicine in which there has been relatively good progress in terms of such translation into regular practice. In this medical specialty, many advances in our increasing scientific understanding of the molecular basis of disease, and patient heterogeneity, have been translated into solutions that benefit patients with specific tumor profiles. Looking across different areas in Oncology, the most successful paradigms that evolved effectively couple the modernization of diagnostics (i.e. the ability to determine tumor subtype based on its biological profile) with the use of targeted therapies (which have been designed for a specific tumor type, or a set of tumor types, with a characteristic biological profile). This personalized health paradigm emphasizes understanding of patient heterogeneity at the level of biological profiles, because it was possible to link diagnostic capability at the level of tumor-derived DNA with its interpretation in terms of the biology that drives the growth and survival of that type of tumor, resulting in impressive improvements of patient outcomes in many tumors (where both tools converge). However, this paradigm has also raised economic sustainability concerns, as tension increases between stakeholders who a) invest in the development of solutions based on this paradigm, and b) those who need to pay for healthcare of tumor patient populations, which are increasingly segmented, with many segments associated with relatively high costs (see above, and Koelsch et al., 2013).
In this renowned area of medicine, many innovations based on this paradigm have advanced quite far in the innovation translation pipeline, leading to practical solutions for global deployment, reimbursement in different healthcare systems, education of healthcare providers, and integration into regular care processes. Considering the effort that is required for such a level of system-wide change in the real world, those successes are indeed quite impressive, keeping in mind, however, that there are many areas of medical need that remain a considerable challenge in oncology, including the phenomenon of tumor recurrence despite short or mid-term effects of targeted therapies.

If we consider to extend this paradigm to other areas of medicine, we need to take into account that tumors have many characteristics that are fundamentally different from many common chronic diseases, complicating the application of exact copies of the approach in non-Oncology areas, apart from some exceptions, such as diseases with a strong genetic component (which tend to be rare). Therefore, we need to learn how to consider the particular characteristics of a disease, at a diagnostic and therapeutic level, as we extend the oncology paradigm of personalized health to other indications. This challenge, so far, has been hard to crack, triggering a 'lessons learned' discourse that can be quite healthy in the context of a possible adoption of the proposed platform. Understanding the very slow progression of many common chronic diseases, from different angles, is part of the scientific challenge, as outlined below.

Another area of medicine that has witnessed much progress in terms of developing a modern approach to the convergence of innovation in diagnosis and therapy is AIDS. Being an infectious disease makes it a case that is quite different from oncology and most chronic diseases, although aspects of the oncology paradigm of targeted therapy and personalization have been re-used here as well. Once the AIDS epidemic was recognized as a major health challenge, relatively fast progress on understanding the key characteristics of viral populations, and the biology of their interactions with host (defense) biology, has enabled the development of highly personalized combination therapy approaches, depending on the DNA level composition of the viral population in that specific patient, at a particular point in time (Lengauer \& Sing, 2006; Lengauer et al., 2014). As in oncology, much of the progress in this area of medicine was catalyzed by technical progress. For example, easier access to relevant omics technology (see below), enables faster, easier and better diagnosis of the state of virus populations, as a basis of therapy personalization. Campaigns for collaborative multi-stakeholder, interdisciplinary solutions for battling this infectious disease have also played an important role in contributing to the relatively fast impact on patient outcomes, although challenges remain, e.g. related to the high costs of many years of combination therapy close to the 'cutting edge' of molecular medicine. Interestingly, note that both areas (oncology and AIDS) have increasingly moved away from the use of single therapies, to a more sophisticated, cutting-edge combination therapy approach that involves the early recognition of disease recurrence. As a consequence, I propose a connected set of successful paradigms from oncology and ADIS as pillars of the platform described below, recognizing that much needs to be learned on how to apply 
those paradigms to chronic diseases with a more limited genetic contribution. In that context, a key question will be to find out where the most meaningful, interpretable and actionable diagnostic signals are, to guide our choice of interventions, based on the patient profile, at a particular stage in disease progression.

\section{Capturing the state of biological systems}

Our ability to study, measure and understand complex biological systems has increased with many new tools and methods - although that doesn't mean that it is easy to put the many different pieces of the puzzle together, in our mind, or in computational models. It is certainly more complex than 'fixing a radio' (Lazebnik, 2002), although the author's thoughtful points about unresolved issues in the biomedical research approach, including the lack of a formal language that helps communities to connect the pieces in such systems, were indeed very helpful, and influential. Enabling technologies in that area includes a maturation of our ability to capture states of biological systems at a more comprehensive level, using genome-wide technologies (or simply 'omics'). Such omics technologies now exist for many different levels of biological systems, including DNA, variants for RNA, protein and metabolite-level system dynamics (i.e. genomics, transcriptomics, proteomics, and metabolomics; Spivey, 2004). Depending on the sample we take and how we process it, omics technologies can generate very rich datasets about the 'expression state' of thousands of molecules in those systems (that are represented by the samples that were taken). However, there are many complex problems in data generation and interpretation as well. Inferring overall 'health states' (see below) from such measurements is possible, but non-trivial, and, at present, still resource intensive (Chen et al., 2012).

Around the year 2000, at about the same time as the hype on the sequencing of the human genome and its ability to revolutionize medicine, there was also much excitement on the promise of such omics technologies (Spivey, 2004), leading to thousands of publications with datasets based on human and non-human samples (e.g. from species that are commonly used as preclinical models of human disease). However, most of those datasets represent 'snapshots' in time, with unclear positioning in terms of disease progression states, exact cellular composition, and other 'metadata' that would help with interpretation and comparison. Now that the first wave of excitement has given way to a second wave that aims to build on lessons learned from the first omics wave, there is increasing awareness about the importance of understanding disease progression, beyond 'snapshots' with limited 'annotation'. This trend is likely to be enabling for the proposed approach, as it helps to connect 'health states' in time, with biology, at a comprehensive level. Looking back at how we handled omics waves could also be tremendously helpful in designing guiding principles for handling technology hypes in general.

\section{Engineering of patient-centric connected health solutions}

Technical advances in a variety of areas, from mobile technology and the widespread use of smartphones, to health-related sensors, machine learning and digitalization of healthcare, are increasingly producing 'real world' impact based on convergence between different technology fields, beyond exciting prototypes, in chronic diseases (Dobkin \& Dorsch, 2011; Kvedar et al., 2016). While the more difficult-to-change and highly regulated healthcare and health innovation sectors are expected to develop more slowly compared to less-regulated industries, e.g. those that can improve products quickly based on consumer-centric feedback loops, there are emerging paradigms with reusability potential. Patient-facing solutions with interfaces for other stakeholders, including healthcare providers, are one of the fastest-moving areas here.

For example, in respiratory diseases, such solutions have connected improved therapy (e.g. new COPD and asthma drugs) with 'real world' data on patient outcomes collected using mobile technology around 'smart inhaler' devices for those drugs, alongside with patient-centric views on smartphones, and the involvement of healthcare providers or clinical trial teams (Bender et al., 2017; Clift, 2016; Perez, 2015). This smart inhaler paradigm for designing "beyond the pill" solutions appears to provide value to multiple stakeholders, as a) the patients get better feedback on how they are doing with the inhaler-based therapy, including the aim to prevent stressful exacerbations, b) healthcare providers have more data to optimize care pathways, c) the developers of relevant drugs get more information on 'real life' settings and problems, enabling faster learning, while d) device developers get better feedback on how to optimize their devices in terms of usability, functionality and other health impacts, and how to connect the engineered systems with other components. Note that the ability to generate such value close to patients' homes, outside classic healthcare settings (e.g. hospitals), is a factor driving excitement in the digital health sector, which has identified the management of chronic diseases as a key challenge and opportunity (for a more comprehensive overview, see Kvedar et al., 2016).

In the context of the proposed platform (below), this and similar patient-centric paradigms fill an important void in the current healthcare and health innovation landscape, as they a) add low cost solutions closer to patients, in their natural environments, minimizing travel to clinics, b) have the potential to contribute diagnostic signals, and c) improve the ability to connect system components, across stakeholders, enabling a more data-driven approach to system-level learning.

\section{Measuring morbidity and disease burden, more globally}

Improvements related to the direct and indirect effects of chronic disease morbidity (and improvements in terms of healthy aging) at population level need to be monitored in a reliable manner, to enable learning based on the impact achieved by different types of candidate solutions. The better we can measure impact, the more efficient our learning process. This needs to be based on a trusted methodology that works in a variety of settings, in different countries, to allow fair comparisons. Recent advances in this area include the "global burden of disease" (GBD) methodology developed by IHME (Institute for Health Metrics and Evaluation), and the framework developed by ICHOM, which establishes a first version of a system for capturing relevant impact. In terms of biomedical innovation, and the key role of clinical studies in validating specific hypotheses in human populations, we can now capture a diversity of patient outcomes, including QoL (Guyatt et al., 1993; Norman et al., 2003) and health-related functions such as mobility (an emerging area enabled by sensors that record different 
kinds of movement patterns, e.g. accelerometry). At the economic sustainability level, measures such as QALY (QoL-adjusted life years) have added the highly debated ability to differentiate among years of life extension with high and low QoL when judging value provided by innovation (Shiroiwa et al., 2010). As a consequence, we now have a basic arsenal of tools to monitor the short and longterm results of solutions we develop, at different levels, in clinical trials, in regular clinical care as well as outside clinical settings. With this, we do have an improved ability to evaluate the impact of system-wide solutions that better connect the fragments, e.g. across the successful paradigms described in this article. However, this does not mean that the current toolbox for measuring outcomes across different settings is perfect and needs no further optimization. It is a very complex topic that will certainly require more innovation and adjustments down the road. At the same time, we can start to pragmatically use what we already have. In that context, multi-morbidity, in a landscape of increasing chronic disease burden, is one of the areas that may benefit from increased attention, with regards to the capture of impact, as well as the combination of several paradigms in relevant solutions.

\section{Open science culture}

An intensive, open debate on the best approach for a particular problem, and open access to data that can help to select among alternative approaches, are features associated with good science. Since the successful paradigm of open source software in informatics has infected an increasing number of areas related to health innovation, including bioinformatics, the screening of chemical libraries, as well as the generation of tools for research on new kinds of drug targets, an intense debate has developed on the role for an extension of those fragmented experiences in particular areas of science into a more comprehensive, interdisciplinary 'open science' approach that includes innovative models for enabling a faster translation of research results to patients (Laverty et al., 2015; Low et al., 2016; Munos, 2010). Indications in which innovative approaches at a similar level of complexity were tested 'end-to-end' have added further fuel to the debate, with the malaria field taking a prominent position among those translational pioneers (Wells et al., 2016). Relevant aspects include a) the openness of raw data, code and algorithms (avoiding 'black box' solutions), which applies to computational as well as experimental protocols, b) 'reproducible research' for enhanced transparency and reproducibility between research groups, c) sharing of data, insights, knowledge, and tools, based on initiatives that provide some structure to data sharing (e.g. Dataverse). Bernard Munos (e.g. Munos, 2010) has proposed that the increasing adoption of such approaches is linked with sustainability in health innovation, considering biomedical complexity. A recent conference in Oxford ("Drug discovery: creating a new ecosytem", 2-3 June 2016) has been able to gather a variety of pioneers in that area. However, while there are many experiences in this growing global community that can help to select a particular open science model for a given medical problem, we are still in the earlier stages of that learning process, in terms of tackling the rather difficult 'valley of death' problem in translation towards patients.

\section{Dealing with real world evidence}

The digitalization of healthcare, as well as technology convergence in non-healthcare areas, are resulting in an increasingly diverse and fragmented landscape of data related to different aspects of health, from hospital records, to claims for reimbursement, to fitness device data, and data produced by patient-centric solutions for chronic diseases as outlined above (Strategy\&, 2015). Such 'real world evidence' is often contrasted with data generated in controlled clinical studies, such as randomized clinical trials that test specific medical hypotheses. Important differences exist, for example, in our ability to make conclusions from either data, based on solid statistics, methodology and theory. In that context, it can be helpful to develop improved capabilities for dealing with real world evidence in a way that is consistent with 'good science' principles, in collaboration with disciplines that have a history in that area, e.g. epidemiology and HEOR (health economy and outcomes research). The example of the "Global Burden of Disease" study of IHME (Lozano et al., 2012), which succeeded in integrating thousands of different real world evidence data sources into an over-arching model that enables a number of analyses, could be helpful in that context.

\section{Business model innovation}

Considering the important role of economy in the health care/ innovation sustainability discussion (with an assumption of limited resources), we can observe that the classic economic model of reimbursement for healthcare actions based on a "fee-for-service" concept, is gradually being replaced by a potentially more sustainable "value-based care" model (EFPIA, 2016). This model is based on the following principles: (i) coordinating around patients all the elements of the care continuum; (ii) shared commitment of all healthcare system players to the outcomes that matter to patients; (iii) generating and tracking data on those outcomes; (iv) benchmarking performance transparently for informing management decisions; and (v) paying for outcomes rather than for inputs and processes. However, this is a complex structural change and therefore unlikely to be an easy transition, so it may take decades before this transformation reaches all aspects of health-related systems globally (with progress being tracked by programs such as the Pharmaceutical Outcomes Research \& Policy Program at University of Washington). In the meantime, pioneering institutions around the world are moving from pilots to organizational change, to become a leader in this important transition, increasing their fitness for the future at an earlier time point, when changes are still easier to manage and resource. One of the exciting opportunities in this area could be an improved ability to better align incentives across stakeholders, based on the above "value-based care" principles, as a basis for more collaborative solution development.

\section{Initial focus indications}

\section{Alzheimer's disease}

Innovation related to this neurological disease is an interesting indication in the context of the proposed systems approach, and platform design, for several reasons:

- It is a common chronic disease with considerable burden on multiple stakeholders, including patients, families, healthcare resources, social care, elderly care, and other areas of society. This burden tends to rise in societies with longer life expectancies (Braak \& Del Tredici, 2015), and is therefore linked with aging populations. 
- It features the typical slow and 'silent' disease progression of many chronic diseases (i.e. sporadic forms of the disease), as tissue damage accumulates over decades in the brain (e.g. as described by Braak \& Del Tredici, 2015, at the level of pathohistology), with patient-specific speed of progression. There are known characteristics of subpopulations of patients with faster progression, e.g. based on genetic predisposition (presenilin families with early onset, APOE4 carriers with medium onset, others with late onset).

- Diagnostic complexity: experience with a very diverse collection of diagnostic tools, including those related to the detection of cognitive decline, molecular biomarkers, imaging biomarkers, histopathology. This could facilitate the iterative optimization of health state models (as proposed below).

- Culture of the field: Many years of disappointing results from clinical trials have resulted in a healthy 'lessons learned' discourse across disciplines, and stakeholders. A history of using computational modeling in the context of biomarkers for disease progression (Haas et al., 2016).

An interesting side effect of the history of disappointing or failed clinical trials in this indication is that it forces the biomedical research community to reconsider their approach and collaborative paradigms for the sake of patients, causing a healthy discussion on advancing the biomedical research community culture, with possible effects in other indications as well, where reusable learnings are generated. For example, this field has suffered from a strong bias for a small set of hypotheses and paradigms, based on particular types of evidence, as a basis for designing and developing therapeutic solutions. The excitement around those hypotheses has resulted in lackluster discussion of things that didn't fit this 'group think', including alternative hypotheses and solutions. Compared to the discourse in this indication in the 1990 s, we can now notice an increasing readiness to learn from this experience.

This neurological disease is a typical chronic disease in the pathobiology sense I discuss below, i.e. there is slowly accumulating tissue damage outpacing regenerative mechanisms, which results in a progressive decline of tissue functions, which then show up as increasingly severe clinical symptoms and patient outcomes are impacted over time. The need to better recognize early stages of disease (Braak \& Del Tredici, 2015), together with innovation in terms of interventions that target the pathobiology of exactly those stages, is now widely seen as the most promising approach for enabling translational progress in the field. This is likely to lead to sophisticated methods for combining diagnostic signals across many system levels, including the cognitive, molecular, imaging and other aspects described above, requiring a platform for improving public reference versions of relevant computational models.

At the same time, there has been good progress in areas related to digital health, e.g. in the early detection of a possible cognitive decline using speech patterns (Morris \& Lundell, 2003), which may add a cheap and easy-to-deploy screening method to the "early stage' diagnostics innovation. The Alzheimer's exemplar may also help in exploring connections between the various aspects of the proposed systems approach and platform. Below, I will propose a road map for developing such a platform, including starting points derived from Alzheimer's disease.

Increasing readiness to design innovative clinical studies is also visible in this indication, related to particular subpopulations and health states, e.g. the APOE4 subpopulation, which carries a higher risk of fast progression towards more severe health states, compared to sporadic cases without such genetic risk factors (Mahley et al., 2009). This may help to close important gaps in the data landscape that prevent progress.

\section{Regenerative medicine, and chronic diseases}

Tissue and organ regeneration principles. We know that, based on knowledge accumulated in scientific fields related to regenerative biology and medicine, a) many animals have an amazing ability to regenerate tissues, organs and limbs (after injury or other damage), and therefore recover in terms of function (i.e. health); and b) that there is considerable evolutionary conservation at the level of the involved biology between humans and non-human vertebrate animals with high regenerative capacity. Discoveries in the past decades in that area have nurtured the hope that a deeper understanding of biomolecular systems involved in tissue, organ and limb regeneration will lead to the development of improved therapeutic and diagnostic solutions for areas of medical need, in which improved regeneration could contribute to better outcomes. In many common chronic diseases we can, during the progression of those diseases in the patient over time, observe a slowly progressing imbalance between accumulating tissue damage, and regenerative mechanisms activated in that tissue as a response to this accumulating damage. I will illustrate this principle using several examples, below, and, in the spirit of the proposed 'systems approach' to chronic diseases, highlight connected aspects from biology, medicine and economy that may enable the development of better solutions.

Chronic liver disease. The liver is a very important organ, contributing to overall health with its many functions related to homeostasis (the ability to keep us within a healthy range, regarding physiological parameters). Depending on our lifestyle, and other factors, such as our genetic profile, liver tissue can be increasingly damaged by different factors, including high consumption levels of alcohol, and an unbalanced (Western) diet (leading to steatohepatitis). This then leads to reduced liver function, with an impact on our body's ability to maintain homeostasis, and therefore health. On the other hand, the liver is also known for its regenerative capacity, a notion that was further reinforced by more recent observations of liver regeneration after the application of antiviral therapies (Zois et al., 2008). In the early stages of such slowly (and silently) accumulating organ damage, the liver may still be able to deal rather well with the repeated insults, and maintain most of the important functions, and therefore overall health. Over time, however, the accumulating damage outweighs the ability to regenerate and maintain organ function, shifting the system towards an unhealthier balance between damage and regeneration. As liver damage increases, and liver function decreases, first clinical symptoms may appear that are often ignored, for a variety of reasons. With a diagnosis of 'late stage liver disease with advanced liver cirrhosis and portal hypertension' by a relevant medical specialist (i.e. a hepatologist), 
a comprehensive reaction of the healthcare system (i.e. a care pathway) with diagnostic and therapeutic aspects is triggered, based on medical guidelines and current understanding of disease progression-related risks). While some patients' lives can be extended through liver transplantation, those who die from liver disease on the transplantation waiting list, waiting for such relief, is unfortunately rising. This adds fuel to a discussion on the need for developing new solutions for this growing medical problem. What we know so far about the different stages of chronic liver disease in such patients can be summarized in the following simplified disease progression model, which includes a heterogeneous mix of causal factors involved in creating the liver tissue damage:

Here, each health state is a stage in disease progression that is characterized by a combination of features that can affect diagnosis, assigning a particular patient to an earlier or later stage in the disease progression (with many consequences related to the clinical management or further diagnostic monitoring of the patient). Based on current knowledge, it seems that, despite the heterogeneity of causal factors involved in creating the relevant tissue damage, there is a clear response pattern of this organ, with limited variation. In other words, once the balance between regeneration and damage has passed a certain level, a rather fixed pattern of progression towards later stages is observed. Some variation is noticed, however, between patients in the time spent between such stages, i.e. we can distinguish 'fast' or 'slow' progression relative to average times, between stages. As such chronic liver disease progression stages have multiple links with the overall health of the patient, in the context of the proposed health state modeling framework they can help to define health states, considering that the same patient may also display other co-morbidities, including other chronic diseases with their own progression stage.

In a similar manner, other chronic diseases result in slowly accumulating tissue damage over the years, until homeostasis and tissue function is affected to a degree that it becomes very difficult to get the patient back to a healthy state with a high QoL. Therefore, the liver disease example may help us define relevant paradigms for understanding health states that consider comorbidity. Unfortunately, such accumulating tissue damage is currently not easy to detect in earlier stages, in real world settings that require a high standard of patient comfort, low cost, ease of use and risk mitigation. For example, repeated invasive sampling of biopsies for assessing the condition of liver tissue in the progression is usually not practiced due to clinical risks associated with biopsy generation. Therefore, we need to develop new solutions that will increase our ability to recognize not only health states and progression stages that have a clear clinical profile, based on currently available tools in the healthcare system, but also help us understand and recognize the health states that are in between very healthy states and the easier-to-recognize advanced stages of chronic diseases. This is a general principle and a grand challenge that no single discipline or stakeholder can fully address on their own.

With this context, what are some of the more interesting interdisciplinary connections involving biology, medicine and economics in developing such new solutions?
- The NAFLD to NASH transition: while many patients have NAFLD, i.e. a fat accumulation in liver that is not likely to be caused by excess consumption of alcohol, but rather by diet and other lifestyle factors (with interesting links to obesity and metabolic syndrome), only a subset of those patients are going to transition into the more serious NASH state in liver disease progression (Calzadilla Bertot \& Adams, 2016). In the NASH stage there is more serious tissue damage, possibly due to an overshooting reaction of the immune systems that is linked with the regenerative response to liver tissue damage. The transition from NAFLD to NASH is loaded with many questions, including the biology of this transition, the best ways to recognize it as early as possible in patients (e.g. Hannah \& Harrison, 2016), and multiple economic consequences, such as the most efficient use of healthcare resources. While much progress has been made in understanding those aspects, a more collaborative approach across stakeholders is increasingly gathering momentum, including projects managed under the IMI umbrella (a framework for multistakeholder collaborations related to health, with publicprivate partnership at its core).

- Effect of co-morbidities: in patients with chronic liver disease, including earlier stages, what are the most relevant co-morbidities that will influence outcomes? While it is easy to see how factors that have a known effect on the regeneration/ damage balance in the liver, e.g. exposure to substances with liver toxicity, will be relevant, epidemiological studies of 'real world data' collected on such patients may reveal additional factors that do not have a known liver tissue relevance. For example, such patients may take drugs (e.g. metformin) on a regular basis that are related to co-morbidities (e.g. diabetes), which interact in a complex manner with the liver disease progression system (e.g. liver functions related to glucose, He et al., 2014). Statistically sound observations in such studies could then lead to investigations into the biology of those drugs in the context of liver disease, with potential effects on clinical management, outcomes and economy that would warrant interdisciplinary collaboration at such interfaces.

- Refining the disease progression model: While it is encouraging to know that there is a highly defined pattern in disease progression in liver diseases, despite the diversity of factors involved in causing tissue damage, there may be heterogeneity in patients that is currently under-appreciated. For example, if we consider the variability that was observed in terms of slow or fast progression between stages, including the risk of developing NASH based on NAFLD, this indicates that we need to learn more about patient heterogeneity and risk of progression, at the biology/medicine interface.

Skin ulcers. The above discussion on chronic liver disease implies a fundamental challenge shared by other chronic diseases, i.e. that we are dealing with slow dynamics in the recognizable transitions between distinct health states. This means that our iterative learning cycle between designing a study, implementing it, sharing the results and designing the next study, combined with the time needed 
to observe sufficient change between health states, leads us to rather long studies that stretch many years. Therefore, fast learning based on short iterative cycles is difficult, apart from problems that can be addressed in shorter timescales. Together with an overall tendency towards short-term approaches including funding, this means that progress on understanding the above problems, including health state refinement, will be hard to accelerate.

With this in mind, let us explore the possibility of finding complementary medical problems related to a) similar interdisciplinary complexity of chronic diseases and b) learning related to health states, c) which would allow us to develop a fast-learning, collaborative network on top of short iterative study cycles and d) a systems approach that facilitates such interdisciplinary exchange. Our example for such a medical problem is again related to the principle of the fateful balance between slowly accumulating tissue damage, and scientific questions in the field of regenerative medicine. When our skin tissues are healthy and have a regenerative capacity within the normal range, we have all experienced how superficially visible wounds usually heal within a few weeks or even days. Once we look a bit closer at this area of tissue regeneration, we can notice a variation in terms of the speed and quality of healing, depending on a variety of factors, such as wound size, shape, depth, use of dressings to promote healing and prevent infection, infection management and so on. In addition, we may have heard about bad outcomes related to wound infection that led to amputations. Based on that common experience, most of us are not used to think of skin wounds as a major medical challenge in the context of the chronic disease challenge. However, if we look even closer, we find that there are many patients with one or more chronic diseases who have considerable problems due to disturbed healing, with surprisingly harsh outcomes linked with how wounds were managed (Hunt et al., 2011; Park et al., 2013). But what is the link between the chronic disease challenge, and this medical problem? And how does it relate to our discussion on systems approaches?

Diabetes complications: Patients with diabetes, in later stages of disease progression, when slowly accumulating tissue damage has reached an advanced stage, may have to deal with a variety of clinical complications, affecting organs such as the eyes, kidney and foot. Complications of the foot typically present themselves clinically as 'diabetic foot ulcer', a type of non-healing, chronic skin wound, to a well-trained expert, such as a specialized wound nurse or physician (Driver et al., 2010; Lim et al., 2017). Unfortunately, many patients carry such dangerous wounds for too long, and therefore have to face unfavorable outcomes, when it's too late to manage the problem with currently available tools. Considering the progress we have made in terms of care coordination and clinical innovation in diabetes, this is one of the remaining problems related to diabetic complications.

Other chronic diseases that affect skin regeneration: To further complicate matters, other chronic diseases also have an effect on such tissue regeneration in the skin after wounding, including venous disease (i.e. leading to 'venous leg ulcers') (Margolis et al., 2002). Proper regeneration of the skin with a full restoration of tissue function (i.e. avoiding a scar with reduced function) requires many cells to do the right thing at the right time in the right context. Once the damage has occurred, there is a wave of signals going through the tissue that triggers that complex and dynamic regenerative response by many cells, including resident cells that go through all kinds of changes, as well as invading cells from the immune systems that arrive on the scene. As a result, a detailed molecular understanding of such skin regeneration is rather difficult, complicating efforts to develop new solutions based on that knowledge.

Towards systems approaches: I mentioned that many chronic diseases, such as liver disease, are difficult to study in terms of disease progression, because of the long timespans involved, which slow down the data-driven, iterative learning cycle. With regards to skin regeneration problems in the context of diabetes and other chronic diseases, the situation is a bit different, because a) changes related to outcomes can be measured in weeks and months, rather than years, b) the fluid produced by open wounds enables omicstype profiling close to the biology of tissue regeneration vs. damage, and c) the most affected tissue is relatively accessible, or easy to monitor, compared to tissues located further inside the body. The combination of those aspects could allow a fast-learning systems approach that combines the paradigms described above. This could then allow connections to be made between:

- Biology of 'wound states', e.g. improving our understanding of the balance between tissue regeneration and damage, how to shift it towards more regeneration, with potential benefits in other chronic diseases

- Clinical profile of 'wound states', e.g. when to intervene to prevent bad outcomes, and how to best integrate new diagnostics into care processes

- Economic profile of 'wound states', e.g. how to achieve good patient outcomes and economic efficiency, considering the secondary and tertiary effects of bad outcome wounds even outside the utilization of healthcare resources

Building a capability for fast learning based on short iterative cycles that enable data-driven approaches, including machine learning and expert-based learning, in the context of an interdisciplinary collaborative network, therefore seems an attractive opportunity in this area of medical need.

\section{Proposed platform}

Design principles guiding the development of the platform I have mentioned the need to a) modernize diagnostics, extending the paradigms developed in leading areas of medical innovation, such as oncology and AIDS; b) connect better across system components that cross disciplines, e.g. medicine, biology and economics of health, e.g. in the context of more patient-centric connected health solutions; c) measure the impact of new PM solutions at that level, in a way that reflects the most relevant outcomes, enabling feedback loops that facilitate faster learning at systems level. But how can we best develop an enabling platform for such systems approaches based on those paradigms, which enables communitybased learning, using open science principles, as well as feedback loops that involve real world evidence? Let us start with the center of the proposed platform, the health states, and their computational modeling across medicine, biology and economics. 
Based on the knowledge we have accumulated in terms of disease progression in Alzheimer's disease and chronic liver disease, I propose to aggregate the medical, biological and economic knowledge across these two indications in a way that allows the extraction of computational and theoretical platform components, as described below (with an eye on later reusability). In a next step, we would test the extension of such a two-indication platform to additional indications (including wound states reflecting skin regeneration), before we would explore the development of an even more comprehensive platform that captures all frequent chronic diseases, their progression stages and complexity at the level of multi-morbidity, for a particular patient. While such a comprehensive platform could have a variety of applications, its use in the design of sequential combinations of interventions, where timing depends on the recognition of a particular health state, is emphasized.

\section{Summary of enabling paradigms}

- A modernization of diagnosis extending (and adapting) paradigms from oncology and AIDS, in parallel with innovation on interventions that build on diagnostic innovation

- Ability of omics profiling technologies to capture aspects of health states at genomic level

- Promoting the design of patient-centric connected health solutions based on technology convergence that provide value to multiple stakeholders, similar to the 'smart inhaler' paradigm

- Build on our increased ability to measure outcomes, morbidity and health at population level, to understand the impact of innovation

- Enabling faster community-based learning through a culture of sharing based on open science, FAIR and reproducible research principles

- Develop solid methodology for dealing with messy 'real world' data, as a complement to more controlled clinical trial data, to understand the patient journey

- Facilitate business model innovation that aligns incentives across stakeholders, facilitating the economic success of more balanced, sustainable approaches

- Extend systems approaches capability through education and community building, see Figure 1 for scope and ambition (health state models may turn out to be only one component in the digital center between stakeholders, but nonetheless a pragmatic focus to learn how to better manage complexity)

\section{Stage 1: Building an initial platform, across two diseases}

If we want to build an initial platform that captures current knowledge in both Alzheimer's disease and chronic liver diseases, how could we get started, based on what exists already? In both indications, we have a relatively good understanding of disease progression states, from a medical, biological and economic point of view. This includes earlier stages of disease (when symptoms tend to be mild, with minimal impact on QoL and healthcare resource usage) to more advanced stages (when symptoms are more severe, with a more dramatic effect on QoL and healthcare resources). At the disease progression level, we can use the following starting points: a) Alzheimer's disease progression theory of Braak \& Del Tredici (2015); b) the review by Pellicoro et al. (2014), summarizing chronic liver disease progression. At the computational level, we can use the following starting points:

Modeling health states. In Alzheimer's disease, there is already a rich history of using computational modeling of distinct health states in the context of disease progression and disease severity, as reviewed by Green (2007). In the words of the author, "Markov models may be particularly useful when a decision problem involves clinical changes, across discrete health states, that are ongoing over time", with such models "representing the course of a disease in terms of mutually exclusive 'health states' and the transitions among them". For example, a 6-month cycle has been used to calculate transition probabilities between states, using clinical trial and epidemiological data. Such computational models are used to assess the value provided by medical interventions, e.g. those that result in a delay of progression towards severe health states. More recent updates on usage of such models, including additional applications, is provided by Green \& Zhang (2016). Based on the small numbers of health states modeled so far, integration of additional data could lead to a population of alternative models with different numbers of health states, initially for Alzheimer's disease only. It may also be necessary to extend the simplistic Markov modeling approach, e.g. considering progress made in projects like the $100 \mathrm{~K}$ cohort (Hood $\&$ Price, 2014) or the Google Baseline study (Piller, 2015). Once an initial version of the computational platform is developed, it could be extended to chronic liver diseases.

Semantic framework. IMI, a renowned multi-stakeholder platform for the development of pre-competitive assets with long-term effects related to medicine, has developed starting points in that area. This includes the Aetionomy project, which enables the representation of complex networks of information, including cause-and-effect relationships, based on the BEL language and the Semantic Web data format $R D F$. Braak and Del Tredici's theory on connections between pathology and clinical symptoms in Alzheimer's (Braak \& Del Tredici, 2015) can be generalized within the platform, in areas that are linked to the above health state models, to capture a slowly progressive tissue damage pathobiology, with regenerative biology context, affecting the dynamics of progression.

\section{Stage 2: Extend to skin regeneration}

Therefore, the stage 1 platform would capture disease progression in two diseases, using Markov models from Alzheimer's as a starting point, with knowledge from liver diseases, a very different indication with different characteristics, enabling the reusability-centric design of health state modeling and semantic framework. Although it would be desirable to then add new knowledge from both indications to the platform, their slowly progressing nature will limit the speed of such iterative optimization. To add faster-learning extensions that benefit the platform development effort, additional areas of medicine will therefore provide a natural focus of stage 2 .

As discussed, skin regeneration as observed in non-healing wounds (skin ulcers) is a promising candidate for stage 2 medical focus areas. Based on existing knowledge on proteins found in wound 
fluid, a fluid similar to but also distinct from blood, panels of candidate protein-level biomarkers could be designed that capture 'wound states'. Wound outcomes linked with such wound states and various 'standard-of-care' interventions could be tracked using nurse-centric Digital health tools that describe progress towards wound closure and healing (i.e. wound outcomes). Such tools could also capture features important for the early detection of potential complications, and facilitate the involvement of relevant experts based on wound states. Smart dressings for wounds that can benefit from negative pressure therapy are under development, within the Horizon 2020 program in Europe, potentially complementing the protein-based monitoring of wound states. In addition, new technologies are available for non-invasive assessments of skin architecture, based on confocal microscopy (Lange-Asschenfeldt et al., 2012). When connected into a comprehensive solution for tracking wounds in wound nurse type settings, such a connected health solution could not only generate valuable real world evidence on a diversity of ulcers, but also advance the ability to recognize and model health states as discussed above. For example, would the platform enable comparisons between innovation efforts in different countries and healthcare settings in a way that is more difficult at present? Testing their ability to model patient journeys in different populations, countries and healthcare settings could help to refine such models in a way that generates a second generation of reference models with an improved ability to capture 'real world' variation, where it matters. The more data become accessible for comparison and optimization, including new data types that extend our knowledge on the biology of different states, as well as diagnostic advances that change the health state recognition part of the models themselves, the more useful such health state models will become, in each iteration of improvement through data-based learning. Connecting them with tools early on that facilitate such iterative optimization will be crucial, including algorithms for the integration of multimodal diagnostic data related to such health states. Special attention should be given to make sure that different patient journey clusters are represented, if differences between those clusters can affect the definition or interpretation of health states, and transitions between health states.

Additional indications. Beyond skin regeneration, additional areas of medicine that are currently not yet identified may become a focus in stage 2 , if they present an opportunity to add faster-learning cycles to the health state learning process, within that platform. Candidate datasets include longitudinal observational human studies that generate data aimed at learning health states and state transitions, including those in early stages of disease, such as the $100 \mathrm{~K}$ project (Hood \& Price, 2014) or the Google Baseline study (Piller, 2015). Other reusable aspects of the platform may also require comparisons beyond those 3 initial indications, e.g. to design a reusable semantic framework around the health state modeling effort.

\section{Stage 3: A platform across many diseases}

Once stage 2 is mature enough, the possibility of extension towards multi-morbidity in chronic disease progression space may present itself. Patterns of multi-morbidity frequently observed in epidemiological data could provide starting points for interdisciplinary focus, in terms of 'real life' health states composed of several chronic disease aspects. Limited resources in elderly care settings could provide an economic aspect, linked to an existing system of cross-indication progression monitoring, including tools for $\mathrm{QoL}$ and risk indicators in those settings.

At that stage, we may be able to approach a more generic and more patient-centric theory of chronic disease progression and health states, across indications. Convergence among the developments discussed above would facilitate its maturation. In particular, diagnostic innovation in slowly progressing chronic diseases would improve our ability to accurately diagnose different stages and variants of disease, including improved understanding of earlier stages of disease that are characterized by a clinically 'silent' progression of tissue damage that increasingly outpaces regenerative, damage control or repair mechanisms. In reference models that capture average disease progression in defined populations, transitions between the states in those models would be calculated using a variety of data, from different diseases, and different types of diagnostic evidence, to capture the characteristics of that population.

Once we have enough information about the most common health states and their reliable recognition, this new capability can be connected with machine learning capabilities that help to refine such models in various settings, based on an initial reference model and some starting conditions that, over time, increasingly fit the features of that particular setting (e.g. the educational and expectation profile of the involved participants, including healthcare providers and patients, as well as established processes, habits and culture). Optimization would be achieved based on feedback loops based on measured outcomes, including patient outcomes, as well as healthcare utilization and other economic aspects, at population level. Reference clusters of patient journeys could be refined, e.g. by adjusting the weights given to particular features in the clustering, filtering for the most predictive features, and including new features not covered in the reference model. Such adaptations should then find their way back to the next generation of reference models as well, so they become easier to adapt to different settings in the next round of optimization.

\section{Applications of the platform}

In later phases of stage 2 , and in stage 3 , various applications of the platform can be envisioned. The examples below are meant for illustration, to encourage participation.

\section{Design of combined interventions}

When single interventions are not sufficient to achieve the desired outcomes, combinations of interventions either delivered at the same time, or in a particular sequence over time (that considers increasingly refined health state recognition capability), will be an interesting option to consider in stage 3. This could mean that we will be increasingly able to iteratively approach a nearoptimal personalized solution for a particular patient, at a particular time in their disease progression, extending the paradigms learned in oncology and AIDS to more indications, and into longitudinal data space. In principle, such value may not need to be limited to therapeutic interventions linked with health states, it could accommodate preventative interventions and even monitoring actions. For example, intervention 'IN1' designed for health state 'HS1' would lead to a subsequent health state HS2, which triggers intervention 


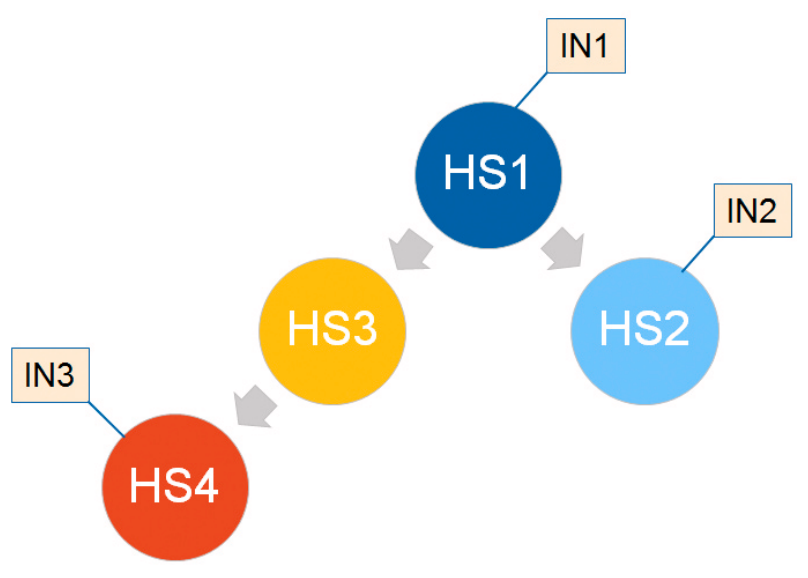

Figure 2. Design of combined interventions, as an application of health state modeling. Health states (HS1-4), which match state definitions in probabilistic Markov models, are connected with interventions (IN1-3), defining the time aspect in the PM vision ("the right intervention for the right patient, at the right time"). Each health state would have annotation in terms of pathobiology, health economics and clinical picture.

'IN2', and so on. After 'HS1' there may be a branching point that, in some patients, leads to another state, 'HS3', which does not match well with intervention 'IN2', but requires monitoring that, once state 'HS4' is reached, triggers intervention 'IN3'. Ideally, the diagnostic recognition of those states HS1-4 would be achieved with a single, reusable diagnostic procedure that is able to differentiate those health states based on non-invasive, low-risk approach (Figure 2)

\section{Patient-facing solutions}

Based on those reference models, user-friendly, patient-facing solutions could be developed, which compare the individual's profile, at a particular time, with the most relevant reference model. If the individual's profile is indicating faster-than-average progression towards one or more disease progression paths, a number of options could be explored, with tools that help monitoring the effect on progression at the level of health states. For example, the beneficial effects of lifestyle changes or therapeutic interventions on that profile could encourage continuation and compliance to relevant guidance. Gamification-related approaches could be useful in exploring aspects related to user engagement, considering expertise from user-centric design.

\section{Clinical studies}

Similar usage may apply to clinical studies, if they span follow-up periods that contain health state transitions. In addition, increased ability to recognize distinct health states could generate hypotheses for new study tools linked with well-established measures and outcomes.

\section{Care coordination}

Coordination of care, including the actions of different healthcare providers, as well as social/elderly care, is a very complex topic. The proposed platform could facilitate such efforts by simplifying the recognition of health states that require actions by specific components in the system, at a particular time, to then follow the effects of those actions on health state transitions more closely. For example, it could facilitate early recognition of worsening condition, complications, and other signals that require attention, and their collaborative interdisciplinary management.

\section{Care pathway redesign}

While (health)care processes are often quite stable over time, once the team and process landscape is up and running, there are periods where such process landscapes are under discussion, to optimize particular outcomes, as well as economic constraints, e.g. for certain patient clusters that show high costs but below-average outcomes. Teams with a history of 'care pathway redesign' could start to engage in the proposed platform, as early as late phases of stage 2. If it is possible to improve the recognition of clinically and economically relevant health states in such settings, care pathway redesign projects would likely be able to extract value from such insights, as they try to determine the best time and mode to intervene in specific types of patient journeys. It could also help them with the exploration of a large range of options for care pathway changes, e.g. in a visual form that supports time-efficient discussion and consensus formation in complex, interdisciplinary groups, based on connected health state diagrams. On the other hand, such collaboration would enable early influence on the design of the proposed platform, to facilitate collaboration at such interfaces, which could become increasingly impactful on both sides over time as health state recognition (via diagnostic tools) and modeling add value to care pathway redesign projects. Such collaborations could therefore benefit both sides, the developers of the proposed platform, as well as the teams involved in care pathway redesign. This includes educational aspects required for such development, with the particular side effect that the platform community gets anchored into 'real life' settings as early as possible. Those focused on disease biology aspects of health states would benefit from such collaborations by improved anchoring of their efforts in 'real life' care settings as well.

\section{Device development}

Institutions involved in device development could benefit from the platform in areas related to chronic disease progression, e.g. in projects aimed at developing smarter, more connected devices that contribute to multi-stakeholder value (similar to 'smart inhalers', see above). For devices linked with therapy application, the platform could help to manage projects, considering challenges related to the different timelines and cultures in therapy and device development, by allowing collaboration without excessive dependency between projects.

\section{Disease biology}

Applications of the platform, related to disease biology, include:

- Enhanced ability to understand translatability of preclinical models, at the level of health state biology

- Improved ability to couple the development of novel therapies with biomarkers related to health states

- Gap analysis at portfolio level, using health states to aggregate project information 


\section{Semantic web}

By closely linking the effort on the development and optimization of health state models with initiatives focused on the representation of semantic aspects of relevant data, the following applications and value can be envisioned:

- Increasing adoption of semantic technologies, for the use of data in models

- Feedback on inconsistencies that help develop the semantic frameworks

- Further development of guiding principles (see below)

Recent progress made in relevant multi-stakeholder communities, such as FORCE11, towards consensus on guiding principles in related areas includes:

- FAIR Guiding Principles, to facilitate data and metadata reuse (Wilkinson et al., 2016)

- Increasing use of semantic web technology for many different types of biomedical data, e.g. RDF versions of EBI resources include diverse objects, from computational models to biosamples, chemicals and gene products (Jupp et al., 2014)

- Increased attention to the importance of capturing reusable metadata, close to data generation, in many institutions. While we are in early stages of connecting across such efforts, convergence with increasing consensus on how to apply FAIR principles will be a key challenge in the coming $5-10$ years.

- Other developments that provide further fuel to such efforts are increasing awareness of the importance of a more consistent implementation of 'reproducible research' principles (Walthemath \& Wolkenhauer, 2016), to restore trust in the results of biomedical research

\section{A few obstacles to keep in mind}

It is not for the lack of motivation, understanding or interest that systems approaches with similarity to the one discussed in this article have not developed towards real world impact, as measured by their contribution to the creation of tangible value to multiple stakeholders, and sustainability/health at systems level. Many obstacles have prevented or at least slowed down progress in that area, including the following factors, which deserve at least a brief discussion:

- Project management experience results in the reduction of complexity, prevention of scope creep and limiting the set of stakeholders involved in decision making, to manage risks associated with the ability to reach agreed deliverables, as well as stakeholder support. Such risk management also means that project leaders are forced to work with what exists, and need to often focus on value for particular stakeholders at the expense of others.

- A tendency to get infected by technology hypes, and other innovation fashions, which often lead to a shift in funding, attention and culture, which reminds us of a 'gold rush', including the 'valley of tears' after the hype, in which models fall apart, predictions are found to be wrong, credibility is lost, widespread frustration about unexpected complexity, and the failure of new wonder drugs (Lazebnik, 2002). As Lazebnik put it, "this stage can be summarized by the paradox that the more facts we learn the less we understand the process we study". If unmanaged, this very human tendency results in an inability to resolve problems at the systems level discussed in this article.

- Attitudes against theory development in science, the role of mathematics, and computational modeling as a tool, further complicate connections with some stakeholders. Such attitudes strongly depend on disciplinary background, highlighting the role of academic education and training in this phenomenon. Life science disciplines, such as biology and medicine, are well-known for a widespread disregard of those aspects, leading to unnecessary tensions with potential contributors from disciplines with stronger emphasis in those areas. An example is the history of omics technologies, where such attitudes and fixed mindsets from the days of a more reductionist "one postdoc, one gene" approach resulted in much waste of research resources due to a lack of experimental design, statistical analysis skills and theoretical background (Micheel et al., 2012). The way we approach problems, and hypes in particular, is at the root of the inability to advance in this area, as highlighted by Lazebnik (2002).

\section{Author contributions}

MR prepared the manuscript, with input on specific topics from experts listed in Acknowledgements.

\section{Competing interests}

The author is an employee of the research organization of a pharmaceutical company (Novartis Pharma AG, Basel, Switzerland). The author acknowledges a bias towards OpenScience principles, as outlined above, which may not reflect the mainstream mindset in his industry.

\section{Grant information}

My employer (Novartis Institutes for Biomedical Research) funded the work on this text.

\section{Acknowledgements}

This text is the result of manifold interactions with the works of others in terms of their publications (not all of them could be listed in References), but also much face-to-face debate on various aspects of the described systems approach. In particular, I would like to acknowledge the contributions from discussions with Dr. Bernard Munos (InnoThink, Indianapolis, USA), Dr. Federico Tortelli (Novartis, Basel), Thomas Brenzikofer (baselarea.swiss, Basel), Dr. Ming Wong (licensed physician, Boston, USA), Dr. Edith Schallmeiner (Roche, Basel), Dr. Thomas Hach (Novartis, Basel), Prof. Torsten Schwede (University of Basel), Dr. Jonas Dorn (Novartis, Basel), Dr. Alex Zhavoronkov (in silico medicine, Baltimore, USA), Dr. Kah-Tong Seow (consultant, Frankfurt, 
Germany), Marco d'Angelantonio (himsa, Brussels, Belgium), Dr. Li Tang (University of Basel), Sascha Kress (Huawei, Zurich), Dr. Stefan Scherer (Novartis, New York), Dr. Evert Luesink (Novartis, Basel), Dr. Florian Nigsch (Novartis, Basel), Dr. Yasuto Tanaka (University of Kobe, Japan), Dr. Vickie Driver (Novartis, Boston, USA), Prof. Keith Harding (University of Cardiff, UK), Dr. Tewis Bouwmeester (Novartis, Basel), Dr. David Gyurko (Kantonspital Aarau), Prof. Christoph R. Meier (University of Basel), Prof. Hinrich Rahmann (University of Hohenheim, Stuttgart, Germany), Prof. Heinz Breer (University of Hohenheim, Stuttgart, Germany), Dr. Hans Widmer (Novartis, Basel), Prof. Russ
Altman (University of Stanford, USA), Dr. Peter Groenen (Actelion, Basel), Dr. Frank Kumli (Ernst \& Young, Basel), Dr. John Lamb (GNF, San Diego), Prof. Michael Krauthammer (Yale University, Connecticut) and Prof. Niko Beerenwinkel (ETH, Basel), and the mentorship I received as a young scientist, including an understanding of 'good science' values, from my academic supervisors (Prof. Harald Rösner, at the University of Hohenheim in Stuttgart, and Prof. Doron Lancet at the Weizmann Institute of Science, in Israel). In a way, this text presents an attempt to find some kind of synthesis between many of those discussions, which were disconnected in so many ways, in time and space.

\section{Supplementary material}

Supplementary File 1: Glossary of terms.

Click here to access the data.

Altman RB: Translational bioinformatics: linking the molecular world to the clinical world. Clin Pharmacol Ther. 2012; 91(6): 994-1000. PubMed Abstract | Publisher Full Text | Free Full Text

Auffray C, Balling R, Barroso I, et al:: Making sense of big data in health research: Towards an EU action plan. Genome Med. 2016; 8(1): 71 PubMed Abstract | Publisher Full Text | Free Full Text

Barker R: 2030 -The future of medicine: avoiding a medical meltdown. Oxford University Press. 2011.

Reference Source

Beckmann JS, Lew D: Reconciling evidence-based medicine and precision medicine in the era of big data: challenges and opportunities. Genome Med. 2016; 8(1): 134-145.

PubMed Abstract | Publisher Full Text | Free Full Text

Bender BG, Chrystyn H, Vrijen B: Smart Pharmaceuticals. In: Health 4.0: How virtualization and big data are revolutionizing healthcare. Edited by Thuemmler $\mathrm{C}$ \& Bai C. ISBN: 978-3-319-47617-9. Springer Int Publish, 2017; 61-90. Publisher Full Text

Bousquet J, Anto JM, Sterk PJ, et al: Systems medicine and integrated care to combat chronic noncommunicable diseases. Genome Med. 2011; 3(7): 43 PubMed Abstract | Publisher Full Text | Free Full Text

Braak H, Del Tredici K: The preclinical phase of the pathological process underlying sporadic Alzheimer's disease. Brain. 2015; 138(Pt 10): 2814-33. PubMed Abstract | Publisher Full Text

Burns LC, Orsini L, L'italien G: Value-based assessment of pharmacodiagnostic testing from early stage development to real-world use. Value Health. 2013; 16(6 Suppl): S16-19.

PubMed Abstract | Publisher Full Tex

Butler D: Translational research: crossing the valley of death. Nature. 2008; 453(7197): 840-842.

PubMed Abstract | Publisher Full Text

Callahan D: Medical progress and global chronic disease: the need for a new model. The Brown Journal of World Affairs. 2013

Reference Source

Calzadilla Bertot L, Adams LA: The Natural Course of Non-Alcoholic Fatty Liver Disease. Int J Mol Sci. 2016; 17(5): pii: E774.

PubMed Abstract | Publisher Full Text | Free Full Text

Carter ED: Making the Blue Zones: Neoliberalism and nudges in public health promotion. Soc Sci Med. 2015; 133: 374-82.

PubMed Abstract | Publisher Full Tex

Chen R, Mias GI, Li-Pook-Than J, et al.: Personal omics profiling reveals dynamic molecular and medical phenotypes. Cell. 2012; 148(6): 1293-1307. PubMed Abstract | Publisher Full Text | Free Full Text

Clift J: Connected asthma: how technology will transform care. Asthma UK Report. 2016.

Reference Source
Cooksey D: A review of UK health research funding. HM Treasury, London; 2006. Reference Source

Dobkin BH, Dorsch A: The promise of mHealth: daily activity monitoring and outcome assessments by wearable sensors. Neurorehabil Neural Repair. 2011 25(9): 788-98.

PubMed Abstract | Publisher Full Text | Free Full Text

Driver VR, Fabbi M, Lavery LA, et al:: The costs of diabetic foot: the economic case for the limb salvage team. J Am Podiatr Med Assoc. 2010; 100(5): 335-41. PubMed Abstract

EFPIA: Healthier future: the case for outcomes-based, sustainable healthcare. European Federation of Pharmaceutical Industries and Associations. 2016.

Reference Source

Flores M, Glusman G, Brogaard K, et al:: P4 medicine: how systems medicine will transform the healthcare sector and society. Per Med. 2013; 10(6): 565-76. PubMed Abstract | Publisher Full Text | Free Full Text

Gerteis J, Izrael D, Deitz D, et al.: Multiple chronic conditions chartbook. AHRQ Publications No. Q14-0038. Agency for Healthcare Research and Quality. 2014. Reference Source

Goodwin B: From control to participation, via a science of qualities. ReVision, 1999; 21: 2-10.

Reference Source

Green C: Modelling disease progression in Alzheimer's disease: a review of modelling methods used for cost-effectiveness analysis. Pharmacoeconomics. 2007; 25(9): 735-50.

PubMed Abstract | Publisher Full Text

Green C, Zhang S: Predicting the progression of Alzheimer's disease dementia: A multidomain health policy model. Alzheimers Dement. 2016; 12(7): 776-85. PubMed Abstract | Publisher Full Text | Free Full Text

Guyatt GH, Feeny $\mathrm{DH}$, Patrick DL: Measuring health-related quality of life. Ann Intern Med. 1993; 118(8): 622-9.

PubMed Abstract | Publisher Full Text

Haas M, Stephenson D, Romero K, et al.: Big data to smart data in Alzheimer's disease: Real-world examples of advanced modeling and simulation.

Alzheimers Dement. 2016; 12(9): 1022-30.

PubMed Abstract | Publisher Full Text

Hammond D: Philosophical and ethical foundations of systems thinking

tripleC. 2005; 3(2): 20-27.

Reference Source

Hawgood S, Hook-Barnard IG, O'Brien TC, et al.: Precision Medicine: Beyond the inflection point. Sci Trans/ Med. 2015; 7(300): 300ps17.

PubMed Abstract | Publisher Full Text

Hannah WN Jr, Harrison SA: Noninvasive imaging methods to determine severity of nonalcoholic fatty liver disease and nonalcoholic steatohepatitis. Hepatology. 2016; 64(6): 2234-43.

PubMed Abstract | Publisher Full Text 
He L, Meng S, Germain-Lee EL, et al.: Potential biomarker of metformin action. $J$ Endocrinol. 2014; 221(3): 363-9.

PubMed Abstract | Publisher Full Text | Free Full Text

Hood L, et al.: 2014-2015 Scientific Strategic Plan. Institute for Systems Biology, Seattle. 2014.

Reference Source

Hood L, Price ND: Promoting wellness and demystifying disease: the $100 \mathrm{~K}$ project. Clinical Omics. 2014; 1(3): 20-23.

Publisher Full Text

Hunt NA, Liu GT, Lavery LA: The economics of limb salvage in diabetes. Plast Reconstr Surg. 2011: 127(Suppl 1): 289S-295S.

PubMed Abstract | Publisher Full Text

Jupp S, Malone J, Bolleman J, et al.: The EBI RDF platform: linked open data for the life sciences. Bioinformatics. 2014; 30(9): 1338-9.

PubMed Abstract | Publisher Full Text | Free Full Text

Kodrič K, Čamernik K, Černe D, et al.: P4 medicine and osteoporosis: a systematic review. Wien Klin Wochenschr. 2016; 128(Suppl 7): 480-491. PubMed Abstract | Publisher Full Tex

Koelsch C, Przewrocka J, Keeling P: Towards a balanced value business model for personalized medicine: an outlook. Pharmacogenomics. 2013; 14(1): 89-102. PubMed Abstract | Publisher Full Text

Kostic A, Phillips R: Precision Medicine as a new paradigm in drug development. Journal of Precision Medicine. 2016.

Reference Source

Kvedar JC, Fogel AL, Elenko $\mathrm{E}$, et al: Digital medicine's march on chronic disease. Nature Biotech. 2016; 34(3): 239-246.

PubMed Abstract | Publisher Full Text

Lange-Asschenfeldt S, Bob A, Terhorst D, et al.: Applicability of confocal lase scanning microscopy for evaluation and monitoring of cutaneous wound

healing. J Biomed Opt. 2012; 17(7): 076016.

PubMed Abstract | Publisher Full Text

Laverty H, Orrling KM, Giordanetto F, et al:: The European lead factory - an experiment in collaborative drug discovery. J Med Dev Sci. 2015; 1(1): 20-33. Reference Source

Lazebnik Y: Can a biologist fix a radio?--Or, what I learned while studying apoptosis. Cancer Cell. 2002; 2(3): 179-82.

PubMed Abstract | Publisher Full Text

Lengauer T, Pfeifer N, Kaiser R: Personalized HIV therapy to control drug resistance. Drug Discov Today Technol. 2014; 11: 57-64.

PubMed Abstract | Publisher Full Tex

Lengauer T, Sing T: Bioinformatics-assisted anti-HIV therapy. Nat Rev Microbiol. 2006; 4(10): 790-97.

PubMed Abstract | Publisher Full Text

im JZ, Ng NS, Thomas C: Prevention and treatment of diabetic foot ulcers.

$J$ R Soc Med 2017: 110(3): 104-109.

PubMed Abstract | Publisher Full Text

Low $\mathrm{E}$, Bountra $\mathrm{C}$, Lee $\mathrm{WH}$ : Accelerating target discovery using pre-competitive open science-patients need faster innovation more than anyone else.

Ecancermedicalscience. 2016; 10: ed57.

PubMed Abstract | Publisher Full Text | Free Full Text

Lozano R, Naghavi M, Foreman $\mathrm{K}$, et al: Global and regional mortality from 235 causes of death for 20 age groups in 1990 and 2010: a systematic analysis for the Global Burden of Disease Study 2010. Lancet. 2012; 380(9859): 2095-128. PubMed Abstract | Publisher Full Text

Mahley RW, Weisgraber KH, Huang Y: Apolipoprotein E: structure determines function, from atherosclerosis to Alzheimer's disease to AIDS. J Lipid Res. 2009; 50(Suppl): S183-8.

PubMed Abstract | Publisher Full Text | Free Full Text

Mahmood SS, Levy D, Vasan RS, et al:: The Framingham Heart Study and the epidemiology of cardiovascular disease: a historical perspective. Lancet. 2014; 383(9921): 999-1008.

PubMed Abstract | Publisher Full Text | Free Full Text

Margolis DJ, Bilker W, Santanna J, et al: Venous leg ulcer: incidence and

prevalence in the elderly. J Am Acad Dermatol. 2002; 46(3): 381-6.

PubMed Abstract | Publisher Full Text

Mathews SC, Pronovost PJ: The need for systems integration in health care. JAMA. 2011; 305(9): 934-935.

PubMed Abstract | Publisher Full Text

Micheel CM, Nass SJ, Omenn GS: Evolution of translational omics: lessons learned and the path forward. Chapter 2: Omics-Based Clinical Discovery: Science, Technology, and Applications. Committee on the Review of OmicsBased Tests for Predicting Patient Outcomes in Clinical Trials; Board on Health Care Services; Board on Health Sciences Policy; Institute of Medicine. 2012.

Reference Source

Morris M, Lundell $\mathrm{J}$ : Ubiquitous computing for cognitive decline: findings from Intel's proactive health research. Intel Research. 2003.

Reference Source

Munos B: Can Open-Source Drug R\&D Repower Pharmaceutical Innovation?
Clin Pharmacol Ther. 2010; 87(5): 534-6.

PubMed Abstract | Publisher Full Text

Munos B: A new look at the most innovative pharma companies, and weather they are sustainable (Forbes Innovation Chatroom). 2016.

Reference Source

Norman GR, Sloan JA, Wyrwich KW: Interpretation of changes in health-related quality of life: the remarkable universality of half a standard deviation. Med Care. 2003; 41(5): 582-92.

PubMed Abstract | Publisher Full Text

Nugent R: Chronic diseases in developing countries: health and economic burdens. Ann NY Acad Sci. 2008; 1136: 70-79.

PubMed Abstract | Publisher Full Text

Park NJ, Allen L, Driver VR: Updating on understanding and managing chronic wounds. Dermathol Ther. 2013; 26(3): 236-56.

PubMed Abstract | Publisher Full Text

Pellicoro A, Ramachandran P, Iredale JP, et al:: Liver fibrosis and repair: immune regulation of wound healing in a solid organ. Nat Rev Immunol. 2014; 14(3):

181-94.

PubMed Abstract | Publisher Full Text

Perez C: Smart inhalers and the future of respiratory health management. $R T$ Magazine. 2015.

Reference Source

Pes GM, Tolu F, Poulain M, et al.: Lifestyle and nutrition related to male

longevity in Sardinia: an ecological study. Nutr Metab Cardiovasc Dis. 2013;

23(3): 212-9.

PubMed Abstract | Publisher Full Tex

Piller C: Google's next big idea: mining health data to prevent disease. STAT

News. 2015

Reference Source

Poste G: Bring on the biomarkers. Nature. 2011; 469(7329): 156-7.

PubMed Abstract | Publisher Full Text

Powell K: All systems go. J Cell Biol. 2004; 165(3): 299-303.

PubMed Abstract | Publisher Full Text | Free Full Text

Pritchard DE, Moeckel F, Susan Villa M, et al:: Strategies for integrating personalized medicine into healthcare practice. Personalized Medicine 2017 ; 14(2): 141-52.

Publisher Full Text

Scholz N: Personalised medicine: the right treatment for the right person at the right time. European Parliament Research Service. 2015; PE 569.009.

Reference Source

Shiroiwa T, Sung YK, Fukuda T, et al:: International survey on willingnessto-pay (WTP) for one additional QALY gained: what is the threshold of cost effectiveness? Health Econ. 2010; 19(4): 422-37.

PubMed Abstract | Publisher Full Text

Spivey A: Systems biology: the big picture. Environ Health Perspect. 2004; 112(16): A938-43.

PubMed Abstract | Free Full Text

Sterling S: Whole systems thinking as a basis for paradigm change in education: explorations in the context of sustainability. PhD thesis at the University of Bath, UK, 2003.

Reference Source

Strategy \& report: Revitalizing pharmaceutical R\&D: The value of real world evidence. 2015

Reference Source

Walthemath D, Wolkenhauer O: How Modeling Standards, Software, and Initiatives Support Reproducibility in Systems Biology and Systems Medicine. IEEE Trans Biomed Eng. 2016; 63(10): 1999-2006.

PubMed Abstract | Publisher Full Text

Wang Y, Xue H, Liu S: Applications of systems science in biomedical research regarding obesity and noncommunicable chronic diseases: opportunities, promise, and challenges. Adv Nutr. 2015; 6(1): 88-95.

PubMed Abstract | Publisher Full Text | Free Full Text

Wehling M: Assessing the translatability of drug projects: what needs to be scored to predict success? Nature Rev Drug Disc. 2009; 8(7): 541-546. PubMed Abstract | Publisher Full Text

Wells TN, Willis $\mathrm{P}$, Burrows $\mathrm{JN}$, et al.: Open data in drug discovery and development: lessons from malaria. Nat Rev Drug Disc. 2016; 15(10): 661-662. PubMed Abstract | Publisher Full Text

Wilckens $\mathrm{T}$ : Machine learning guided precision trials for major chronic debilitating diseases. HBR Forum. 2016.

Reference Source

Wilkinson MD, Dumontier M, Aalbersberg IJ, et al.: The FAIR Guiding Principles for scientific data management and stewardship. Sci Data. 2016; 3: 160018. PubMed Abstract | Publisher Full Text | Free Full Text

Yang X, Wu X, Hao HL, et al.: Mechanisms and assessment of water eutrophication. J Zhejiang Univ Sci B. 2008; 9(3): 197-209.

PubMed Abstract | Publisher Full Text | Free Full Text

Zois $\mathrm{CD}$, Baltayiannis $\mathrm{GH}$, Karayiannis $\mathrm{P}$, et al.: Systematic review: hepatic fibrosis - regression with therapy. Aliment Pharmacol Ther. 2008; 28(10): 1175-87. PubMed Abstract | Publisher Full Text 


\section{Open Peer Review}

\section{Current Peer Review Status:}

\section{Version 1}

Reviewer Report 13 June 2017

https://doi.org/10.5256/f1000research.11956.r21818

(C) 2017 Auffray C et al. This is an open access peer review report distributed under the terms of the Creative Commons Attribution License, which permits unrestricted use, distribution, and reproduction in any medium, provided the original work is properly cited.

\section{Charles Auffray}

European Institute for Systems Biology \& Medicine, Université de Lyon, Lyon, 69007 CEDEX 07, France

\section{Bertrand De Meulder}

European Institute for Systems Biology \& Medicine, Université de Lyon, Lyon, 69366 CEDEX 07, France

\section{Manlio Vinciguerra}

Center for Translational Medicine, International Clinical Research Center (FNUSA-ICRC), Brno, Czech Republic

This excellent paper proposes to learn from progresses in leading areas of medicine innovation (AIDS, oncology, Alzheimer's disease) to extend them to other chronic diseases using system approaches. Representing health states with the aim of designing the right intervention for the right patient at the right time and dose should allow reiterative approaches in longitudinal studies. The paper focus on modernization of diagnosis (omics etc), technologies/digital health and biological/clinical (biomarkers) and and economic aspects of the disease.

A comment that can be done to the paper, only partially covered by other reviewers, is that it tries to cover a too large subject, which makes it difficult to read; while avoiding, in our opinion, some crucial points.

It is also of note that the readability of the paper could be greatly improved by focusing on the key messages; the author tends to drift off topic and his lyrical musings, although interesting, add a lot of weight to an already large paper.

We would omit/reduce the sections on Open science culture and Business model innovation, Semantic web.

We would also reduce the section on systems approaches, that are already well covered in the manuscripts cited in the text. 
Blue zones, islands of longevity: the author provocatively states how it is possible to translate the information that being a shepherd who eats barley and live in the mountain to the general population. Genetics of centenarians is a very important research field. We now know that specific mutations associated to IGF-1 are enriched only in centenarians. We can also learn a lot in terms of food habits and nutraceuticals from extreme long-lived healthy people. Diet, moderate intake or animal proteins. Exercise. Prevention.

Family ties: Emma Morano Martinuzzi, the longest lived person on the planet recently passed away aged 117.5 years old. Emma had something that ALL long lived healthy people share: extensive family social network of support. A true army of offspring, grandchildren, cousins etc. Emma was never alone. In modern Western societies old people tend to isolate and die alone in their houses.

Loneliness is notoriously associated to lowering immune defenses, depression and chronic diseases. It is all linked.

An important section of the paper should be dedicated to governmental/social policies not to leave our elderly to age and die alone (robots are not a solution), which happens less in the Eastern world or in more archaic societies.

Medical knowledge, conceptual framework, technological advances and semantic categorization of stakeholders interactions are useless exercises without removing the elephant in the room: the sentimental and civil value we give to our elderly.

Minor comment: in page 5, the author links the development of human civilization with the eutrophication of waters. We think that linking eutrophication with human activity should not be automatic. Eutrophication can be the product of a natural process, such as unusually high temperatures, modification of the hydrogeology of the ecosystem, natural erosion process of rich soils causing a sudden rise in availability of certain nutrients...

Is the topic of the opinion article discussed accurately in the context of the current literature?

Partly

Are all factual statements correct and adequately supported by citations? Yes

Are arguments sufficiently supported by evidence from the published literature? Yes

Are the conclusions drawn balanced and justified on the basis of the presented arguments? Partly

Competing Interests: No competing interests were disclosed.

We confirm that we have read this submission and believe that we have an appropriate level of expertise to confirm that it is of an acceptable scientific standard, however we have 
significant reservations, as outlined above.

Reviewer Report 30 May 2017

https://doi.org/10.5256/f1000research.11956.r21819

(C) 2017 Beckmann J. This is an open access peer review report distributed under the terms of the Creative Commons Attribution License, which permits unrestricted use, distribution, and reproduction in any medium, provided the original work is properly cited.

\section{Jacques S. Beckmann}

Université de Lausanne, Lausanne, Switzerland

There is a general excitement about the perspectives of improved medical care and therapies afforded by the recent emergence of cost-effective high-throughput disruptive technologies, allowing the generation and computational analyses of massive amounts of clinically-relevant data and their transformation into useful information. This hype is evidenced by the abounding scientific literature on these subjects. The current manuscript summarizes the field approaching it from the perspective of pharma industries and focusing on chronic diseases.

I read it more as representing as a first step, promoting a dialogue between the interested stakeholders, triggering reactions in order to eventually catalyse or crystalize the convergence of synergistic approaches.

The manuscript lists numerous elements of discussion and consideration. This list is obviously incomplete, each reader may have his own favourite points to add. But this is also the purpose of this article (to which I could add my pet projects or thoughts).

Indeed, in many aspects, this opening call to "put all the pieces of the puzzle together" may suffer from being yet incomplete, partially superficial or presenting an overly simplified interpretation; it may elicit disagreements on particular points. Yet, these limitations essentially reflect the uncertainties in which this field is currently navigating.

As such the manuscript warrants publication in F1000, a forum where such discussions are encouraged.

Specific comments:

I like the wink to the tension between civilization, lifestyles and new technologies or what is referred to as "the other side of the coin". Future discussions might also consider the potential risk of a further dramatic narrowing of the cultural, ethnic, species and environmental diversity, the consequences of which we don't fathom fully as yet (although there have been numerous historical precedents, also illustrated in Y Harari's

book).

I would encourage further discussions on the deer necessity for the implementation of standardized, consistent nomenclatures and ontologies allowing, as suggested, cross-disease interactive channels. This is a real challenge as clinical data are much more complex and 
heterogeneous than lab data. To this we must add additional obstacles, when considering patientcentred involvement: these concern, among others, language (and cultural) barriers, the abundance of non-clinically approved monitoring devices and the heterogeneity in patients' verbal description of their status.

Minor item:

Correct ADIS to AIDS (under Modernization of diagnosis and personalized therapy)

\section{Is the topic of the opinion article discussed accurately in the context of the current literature? \\ Yes}

Are all factual statements correct and adequately supported by citations?

Yes

Are arguments sufficiently supported by evidence from the published literature?

Yes

Are the conclusions drawn balanced and justified on the basis of the presented arguments? Yes

Competing Interests: No competing interests were disclosed.

Reviewer Expertise: genetics, precision medicine

I confirm that I have read this submission and believe that I have an appropriate level of expertise to confirm that it is of an acceptable scientific standard.

Reviewer Report 04 May 2017

https://doi.org/10.5256/f1000research.11956.r22492

(c) 2017 Hooft van Huijsduijnen R. This is an open access peer review report distributed under the terms of the Creative Commons Attribution License, which permits unrestricted use, distribution, and reproduction in any medium, provided the original work is properly cited.

\section{Rob Hooft van Huijsduijnen}

Independent consultant, Geneva, Switzerland

The author proposes to better exploit the vast and varied datasets that are being generated around -specifically- chronic diseases to break these down in a set of 'health states' that can be used to guide improved prevention and treatment. Implementing such a view ('platform') requires the breakdown of barriers to Open Access and (paraphrasing) a revolution in health economics.

These ideas are familiar to many who have spent time in the Pharma industry and sometimes lead 
to heated discussions. This reviewer, for one, subscribes to the majority of the views that are expressed here.

The only real criticism I have is that the piece attempts to cover a lot of ground, and that the ideas could be presented in a more easily digestible way. Some very complex issues are lightly touched upon (the added Glossary is quite helpful). There are few specific, actionable recommendationsthe way I read this is to prepare the mind-set for how better to tackle the great medical challenges of the $21^{\text {st }}$ Century. In itself that is OK if the message is clear.

Some of the language is awkward- I would not focus on boring 'health states', for, in analogy with Tolstoy, 'all happy families are alike'. I guess what is actually meant here is 'disease states', and I would make that 'disease stages' to convey the dynamics of healing and worsening disease.

Overall, I had severe difficulties reading the title and abstract to make out what the article is about (dawned upon me much later). I initially half-expected this to be about holistic or anthroposophical medicine, or equivalent nonsense. In terms of informing and capturing the reader's attention I feel these sections can (and must) be much improved. The Journal Nature typically throws in 'Boxes' where complex concepts are explained. If the author is to reach a broad readership that includes academics this might be a good idea.

The section of a changing medical landscape towards chronical diseases is interesting but the author does not specifically say why Pharma is inept to deal with these. The casual reader might ask here- "so what?" It is not clear how the 'Islands of healthy aging' fits in; I guess it is to say that genes and environment play important roles, and this analysis promotes the article's system approaches but then we are told that "Other conclusions may be valid as well, and it can be difficult to choose among the alternative conclusions, to inform action."- suggesting the problem is in fact intractable.

Examples are given where modern systems biology has made good progress with improved diagnostics and interventions: oncology and AIDS. Fair enough, but these are diseases where the 'enemy' is crystal-clear. These disease states can almost be reduced simply to the metastatic tumour mass resp. virus load- it is not obvious the elegant solutions in this area are transferrable to 'regenerative medicine', the focus of the article. At some point it is said that "..biology of their [AIDS] interactions with host (defense) biology, has enabled the development of highly personalized combination therapy approaches". I disagree- almost all HIV drugs I know simply target viral enzymes or HIV's binding to CCR5. I just fail to see how these successes illustrate how we can tackle Alzheimer's, diabetes and similar diseases. The article would really gain from better suggesting how the proposed approach could help us in the right direction here.

What I like however is the author's suggestion to be comprehensive in evaluating datasets that may affect the dynamics in disease stages. Also, his suggestion for better, global monitoring and standardized data collection merits follow-up. In this context the NHS could be cited, which is in a position where it can, and actually does such things. Also, I agree with the insight that we must let go of our ambition to fully understand systems. Some people understand how computers work, and some computers play better chess than any human being- likewise we will eventually understand the nuts and bolts of life- but remain unable to predict how humans will behave.

I am a bit surprised that biomarkers are mainly presented as helping to understand 'disease 
states'. For me the main asset is that they can accelerate the evaluation of experimental drugs in patient (proxy outcomes). I am not too familiar with P4 and 'personalized medicine' for chronic diseases. I think the goal for now is to identify any treatment for, again, e.g., Alzheimer's for any category of patients- in my time we called that 'patient stratification'- but renaming elusive targets may provide some relief to frustration...

Another topic that could easily be expanded into a doorstopper is combinatorial interventions. This reviewer fully agrees that the concept of treating complex diseases with a single molecule is outrageous. However, evaluating multiple combinations in a factorial clinical trial design is just impossible, to mention just one problem.

The other topic that is (too) lightly touched upon is the disconnect between health care investment, outcomes and payback (health economics). I haven't seen much "value-based care", with country-by-country (differential) pricing and too many clinical trials where NCEs are tested against placebos rather than the best existing care. Pharma (and Biotech) companies still behave as if they are just competing against each other, zero-sum-wise, spending more on marketing than on R\&D in the process. As this article implies, they should rather be playing the game where they try to guess the cards that Mother Nature holds- and this requires cooperation. It is refreshing (and not wholly surprising) that such a proposal emerges from Novartis, one of the more enlightened Pharma Companies (no commercial interests with this reviewer).

Many of the points raised above are intended to improve the important messages in this article and are to be seen as constructive criticism.

Is the topic of the opinion article discussed accurately in the context of the current literature?

Yes

Are all factual statements correct and adequately supported by citations?

Yes

Are arguments sufficiently supported by evidence from the published literature? Yes

Are the conclusions drawn balanced and justified on the basis of the presented arguments? Yes

Competing Interests: No competing interests were disclosed.

Reviewer Expertise: Drug discovery, molecular biology, genomics, infectious diseases, neurodegenerative diseases

I confirm that I have read this submission and believe that I have an appropriate level of expertise to confirm that it is of an acceptable scientific standard. 
Michael Rebhan, Novartis Institutes for Biomedical Research, Basel, Switzerland

The referee has provided a very interesting, constructive, in-depth review of this article, identifying several aspects of the text that warrant the generation of a 2 nd version of the paper with the aim of reduced ambiguity for specific aspects. As author my intention is to work with several co-authors who have relevant expertise on those improvements in the next months, so the community has a more useful reference text for a discussion of those aspects.

In the meantime, let me comment on some of his points right away, to further stimulate discussion:

1. Implementing the platform requires the breakdown of barriers to Open Access, and a revolution in health economics: Agreed, this is a tough challenge, for a variety of reasons but what is the alternative we have as a society struggling with rapidly rising chronic disease burden, and healthcare costs? While it is clear that the current health economics in real life is quite different from the approach described in the paper, many pioneers increasingly agree that value-based models are an important part of a more sustainable approach we need to develop, and that fragmented discipline-specific innovation efforts will not get us there. Implementing it however is hard work, extremely complex, and likely an iterative learning process. It is not developing as fast as many of us would hope, on the ground, in many areas; but there is progress, e.g. look at the work of BD4BO in IMI, which helps to create an improved, practical foundation for such efforts. It is clear that we need to develop a more sustainable system, and that it is difficult to get fast traction in the real world, to a point where many of us may just give up once they know the true complexity of the challenge. But what is the alternative? Just keep talented innovators focused on easierto-handle fragments with short-term effect that do not connect at system sustainability level? The vision of the paper may seem overly ambitious and idealistic to many short-term focused pragmatists, but I wonder if a bit more focus on long-term oriented, collaborative systems approaches such as what is proposed in this paper would create a more healthy balance in our health innovation ecosystem. Think of it as a portfolio approach to short and long term aspects, with the right balance between both.

See http://www.efpia.eu/topics/innovation/outcomes

2. There are few specific, actionable recommendations, it's more about preparing the mindset for learning how to better tackle the great medical challenges of the 21st century: I do not agree with this statement. Did the referee notice the proposed roadmap for developing a health state modeling platform, towards the end of the paper (stages 1-3)? The way it is described there it could be implemented with limited resources, in a few years, with some refinement along the way as the community builds, and data are collected on what works best. On the other hand, preparing mindsets to focus more on long-term sustainability may be a value nonetheless?

3. Oncology and AIDS as examples: Partly agree. The referee comments that it is not obvious how the elegant solutions in those indications, where the root of the problem is more defined and easier to capture (e.g. at pathobiological level), can be transferred to 
chronic diseases and regenerative medicine. I agree that we cannot simply take the exact approaches that were used there, copy and paste them, and then apply them 'as is' in the indications described in the paper. As we accumulate more data on health states, learn how to represent and optimize such states, and build models for different uses, we need to learn which aspects and variants of those paradigms can be applied to which problems, and where the limitations are of a particular paradigm in terms of its application. There is no silver bullet that solves all problems, we all know that. Simply getting everyone's DNA and looking for signals there is not likely to be enough for chronic diseases with complex temporal change patterns, even if we do a lot of it in very large populations. Considering the proposed focus on the aggregation of longitudinal human data that can be simplified as health state models we need to first find out where the most relevant signals are that we should focus on.

4. Why is Pharma inept to deal with the changing medical landscape toward chronical diseases? This is an interesting question, which may best be addressed in a separate, followup paper, as it is quite complex. The intention of this paper is NOT to focus on a Pharma perspective. Instead, the intention is to zoom out and look at the problem from a more neutral but comprehensive perspective which is more likely to be relevant for different stakeholders, as described in Fig. 1. While the author cannot claim neutrality based on affiliation, a serious effort was made to avoid a Pharma bias in the text, and provide a more balanced perspective. The proposed platform for health state modeling can only work as an open crystallization point in the community if it achieves a healthy balance of interests (Fig. 1), but this certainly requires a transition from an atmosphere of blaming each other for failures at systems level, to true collaboration around more constructive and sustainable approaches. There are important fundamental problems that are hard to tackle, some of them mentioned by this referee, which require a new constructive culture that transcends institutions, interest groups and mindsets. Nobody said it will be easy. I think there are plenty of people in Pharma and other places who are ready to engage, not only in Novartis. Again, what is the alternative for society? Business as usual?

5. Biomarkers, health vs. disease states and patient stratification: The referee has many interesting points here, as the paper offers a somewhat unorthodox view of the role of biomarkers, in the context of patient stratification and disease progression. In the text, the way 'biomarkers' are discussed in terms of their relevance for the proposed health state modeling platform, as this is the focus of the text. Of course they will have additional uses and meaning, in other contexts. On the other hand, health states could also be defined in a way that not only relies on data related to biomarkers in a narrow sense, but also other objectively measurable signals that are usually not considered biomarkers. Once the health states have sufficient detail in terms of the most relevant disease biology states, linked with a clinical and economic profile, they could be seen as models that provide actionable interpretations and visualizations for combinations of biomarkers combined with other signals. The preference for the term 'health states' over 'disease states' comes from the idea that we still do not know enough about transitions between health and disease, and the earlier stages of many diseases. However, it is possible that therapeutic or preventative interventions in those earlier stages are likely to be more cost-effective and provide better patient value than later interventions. The way we look at disease and medicine currently is often focused on later stages with a strong phenotype that is visible in a classical clinical 
setting, with a higher hurdle for interventions to make a difference, and less emphasis on screening and preventative approaches. Again, this takes us back to economic reality in health, which may need a bit of adjustment, see above.

Competing Interests: See the article

Reviewer Report 24 April 2017

https://doi.org/10.5256/f1000research.11956.r21975

(C) 2017 Goldman M. This is an open access peer review report distributed under the terms of the Creative Commons Attribution License, which permits unrestricted use, distribution, and reproduction in any medium, provided the original work is properly cited.

\section{Michel Goldman}

Institute for Interdisciplinary Innovation in Healthcare, Université Libre de Bruxelles, Brussels, Belgium

Excellent article that will be inspirational for shaping the future of medicine and healthcare. The systems approach should indeed revolutionise the approach of chronic disorders. The authors rightly integrate the importance of patient-centricity and digital health to translate precision medicine into standard of care. The F1000Research Open Science is a very appropriate vehicle for dissemination of this innovative vision.

Is the topic of the opinion article discussed accurately in the context of the current literature?

Yes

Are all factual statements correct and adequately supported by citations?

Yes

Are arguments sufficiently supported by evidence from the published literature? Yes

Are the conclusions drawn balanced and justified on the basis of the presented arguments? Yes

Competing Interests: No competing interests were disclosed.

Reviewer Expertise: Strategies for therapeutic innovation

I confirm that I have read this submission and believe that I have an appropriate level of expertise to confirm that it is of an acceptable scientific standard. 


\section{Comments on this article}

\section{Version 1}

Author Response 19 Jun 2017

Michael Rebhan, Novartis Institutes for Biomedical Research, Basel, Switzerland

A 2 nd version of this article is being prepared. Contact me if you have any questions or suggestions, or feel free to use the commenting features at F1000Research.

Best wishes, Michael Rebhan (michael.rebhan@novartis.com)

Competing Interests: No competing interests were disclosed.

The benefits of publishing with F1000Research:

- Your article is published within days, with no editorial bias

- You can publish traditional articles, null/negative results, case reports, data notes and more

- The peer review process is transparent and collaborative

- Your article is indexed in PubMed after passing peer review

- Dedicated customer support at every stage

For pre-submission enquiries, contact research@f1000.com 